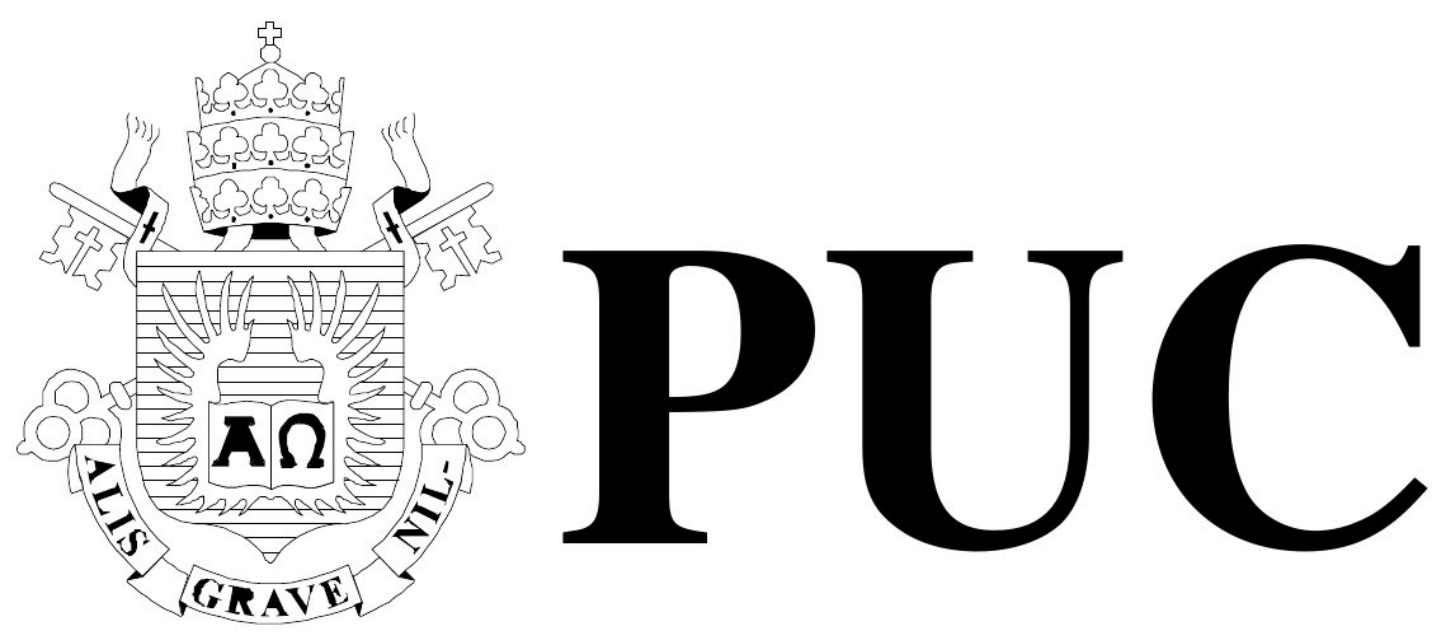

ISSN 0103-9741

Monografias em Ciência da Computação

$n^{\circ} 12 / 12$

CÉU: Embedded, Safe, and Reactive Programming

Francisco Sant'Anna

Noemi de La Rocque Rodriguez

Roberto lerusalimschy

Departamento de Informática

PONTIFÍCIA UNIVERSIDADE CATÓLICA DO RIO DE JANEIRO

RUA MARQUÊS DE SÃO VICENTE, 225 - CEP 22451-900

RIO DE JANEIRO - BRASIL 


\title{
CÉU: Embedded, Safe, and Reactive Programming
}

\author{
Francisco Sant'Anna Noemi de La Rocque Rodriguez \\ Roberto lerusalimschy
}

\begin{abstract}
fsantanna@inf.puc-rio.br, noemi@inf.puc-rio.br ,roberto@inf.puc-rio.br
\end{abstract}

\begin{abstract}
CÉU is a programming language that unifies the features found in dataflow and imperative synchronous reactive languages, offering a high-level and safe alternative to event-driven and multithreaded systems for embedded systems.
\end{abstract}

CÉU supports concurrent lines of execution that run in time steps and are allowed to share variables. However, the synchronous and static nature of CÉU enables a compile time analysis that can enforce deterministic and memory-safe programs.

CÉU also introduces first-class support for "wall-clock" time (i.e. time from the real world), and offers seamless integration with $C$ and simulation of programs in the language itself.

The CÉU compiler generates single-threaded code comparable to handcrafted $C$ programs in terms of size and portability.

Keywords: Concurrency, Synchronous, Determinism, Static Analysis, Embedded Systems

Resumo. CÉU é uma linguagem que unifica os estilos dataflow e imperativo das linguagens síncronas reativas, oferecendo uma alternativa segura e de alto nível aos modelos orientados a eventos e multithreaded para sistemas embarcados.

CÉU suporta linhas de execução concorrentes que avançam em passos de tempo e podem compartilhar variáveis. No entanto, a natureza síncrona e estática de CÉU viabiliza uma análise em tempo de compilação que assegura programas determinísticos.

CÉU também introduz suporte de primeira classe para wall-clock time (i.e. tempo do mundo real) e oferece integração com $C$ e simulação de programas na própria linguagem.

O compilador de CÉU gera código single-threaded comparável a programas $C$ em termos de tamanho e portabilidade.

Palavras-chave: Concorrência, Síncrono, Determinismo, Análise Estática, Sistemas Embarcados. 


\section{In charge of publications :}

Rosane Teles Lins Castilho Assessoria de Biblioteca, Documentação e Informação PUC-Rio Departamento de Informática

Rua Marquês de São Vicente, 225 - Gávea 22451-900 Rio de Janeiro RJ Brasil

Tel. +55 21 3527-1516 Fax: +55 21 3527-1530

E-mail: bib-di@inf.puc-rio.br

Web site: http://bib-di.inf.puc-rio.br/techreports/ 


\section{Contents}

\begin{tabular}{lll}
\hline & Introduction & 1
\end{tabular}

2 The Language CÉU 2

2.1 Parallel compositions . . . . . . . . . . . . . . . . . . . 5

2.2 Internal events \& Dataflow support . . . . . . . . . . . . . . . . . . 6

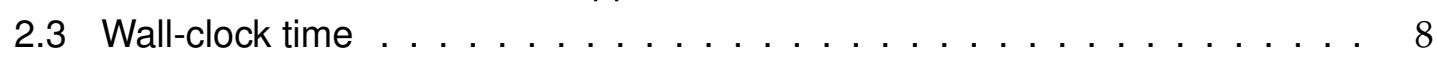

2.4 Integration with $C \ldots \ldots \ldots \ldots \ldots$

2.5 Bounded execution . . . . . . . . . . . . . . . . . . . . . . . . . 9

2.6 Determinism . . . . . . . . . . . . . . . . . . . . . . . . . . . . . 10

2.7 Asynchronous execution . . . . . . . . . . . . . . . . . . . . . . . . . 13

2.8 Simulation in CÉu $\ldots \ldots \ldots \ldots \ldots \ldots . \ldots \ldots$

2.9 GALS execution $\ldots \ldots \ldots \ldots \ldots$

\begin{tabular}{lll}
\hline & Demo applications & 16
\end{tabular}

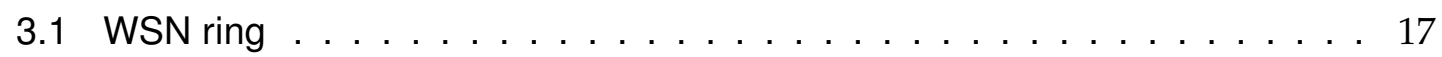

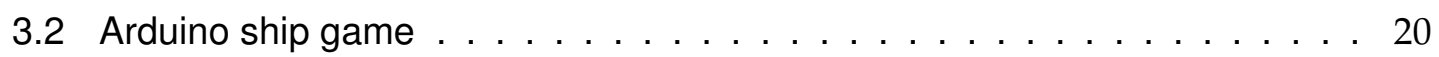

3.3 SDL game simulation $\ldots \ldots \ldots \ldots \ldots \ldots$

4 Implementation of CÉU $\quad 28$

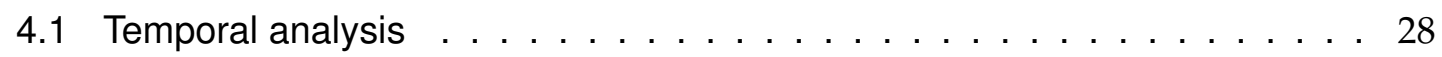

4.2 Memory layout . . . . . . . . . . . . . . . . . . . . . . . . . . . . . . . . . . 29

4.3 Gate allocation . . . . . . . . . . . . . . . . . . . . . . . . . . . . . . . . . . 29

4.4 Code generation . . . . . . . . . . . . . . . . . . . . . 30

4.5 Reactive execution . . . . . . . . . . . . . . . . . . . . . . . . . 31

4.6 Evaluation . . . . . . . . . . . . . . . . . . . . . . . . . . . . . 32

\begin{tabular}{lll}
\hline 5 & Related work & $\mathbf{3 4}$
\end{tabular}

5.1 Synchronous model . . . . . . . . . . . . . . . . . . . . 34

5.2 Asynchronous model . . . . . . . . . . . . . . . . . . . . . . 36

\begin{tabular}{lll}
\hline Conclusion & 36
\end{tabular}

\begin{tabular}{ll}
\hline References & 37
\end{tabular}

\begin{tabular}{|l|l|}
\hline A Syntax of CÉU & 39
\end{tabular} 


\section{Introduction}

Embedded systems combine hardware, software, and possibly mechanical devices to perform a specific dedicated task. They differ from general-purpose systems, which are designed with flexibility in mind and encompass a multitude of applications in a single system. Examples of embedded systems range from simple MP3 players to complex flyby-wire avionic systems. Usually they have a very low tolerance to faults, are constrained in memory and processing, and must conform with real-time requirements.

Embedded systems are essentially reactive as they interact permanently with the surrounding environment through input and output devices (e.g. buttons, timers, touch displays, etc.).

Software for embedded systems is usually developed in $C$, and the addition of a realtime operating system may extend it with preemptive and/or cooperative multithreading $(M T)$. However, concurrency in $C$ requires a low-level exercise related to the life cycle of activities (i.e. creating, starting, and destroying threads), besides extra efforts for explicit scheduling (in cooperative MT) and manual synchronization (in preemptive MT). Furthermore, these models lack safety warranties, given that cooperative MT is susceptible to unbounded execution, while preemptive $M T$ is subject to race conditions and deadlocks.

An established alternative to $C$ in the field of safety-critical embedded systems are reactive synchronous languages [1]. Two major styles of synchronous languages have evolved: in the control-imperative style (e.g. Esterel [2]), programs are structured with control flow primitives, such as parallelism, repetition, and preemption; in the dataflowdeclarative style (e.g. Lustre [3]), programs can be seen as graphs of values, in which a change to a value is propagated through its dependencies without explicit programming.

We believe that embedded systems programming can benefit from a new language that reconciles both reactive synchronous styles, while preserving typical $C$ features that programmers are familiarized, such as shared memory concurrency.

In this work, we present CÉU, a reactive language targeting embedded systems that unifies both imperative and dataflow synchronous programming styles. CÉU is based on a small set of reactive control primitives similar in functionality to Esterel's [2]. On top of this kernel, CÉU provides disciplined side effects, which together with internal events enable dataflow capabilities to the language.

Besides offering a high-level reactive programming model, a primeval goal of CÉU is to ensure the correctness of programs through safety warranties. CÉU relies on a compiletime analysis to detect unbounded loops and concurrent access to variables. The static analysis precludes any dynamic support in the language, such as memory allocation, a call stack, and dynamic loading. However, this trade-off seems to be favorable in the context of embedded systems, as dynamic features are discouraged due to resource limitations and safety requirements.

CÉU has an open source implementation ${ }^{1}$ targeted at highly constrained embedded platforms, such as Arduind ${ }^{2}$ and wireless sensor nodes (e.g. mica $2^{3}$ ). The current memory footprint of the CÉU runtime is around 3Kbytes of ROM and 100bytes of RAM on a 16-bit platform.

${ }^{1}$ http://www. ceu-lang.org

2 http://www.arduino.cc

3 http://www.xbow.com 
This paper is organized as follows. In Section 2, we introduce CÉU: its synchronous execution model, parallel compositions, dataflow support, first class wall-clock time, integration with $C$, safety warranties, and asynchronous execution. In Section 3 , we demonstrate the applicability of CÉU with three demo applications in different scenarios, exploring the programming style promoted by the language. In Section 4, we discuss the implementation of CÉU. In Section 5, we compare CÉU with common approaches for programming embedded systems. In Section 6, we make final remarks and conclude the paper.

\section{The Language CÉU}

CÉU is a concurrent language in which multiple lines of execution-known as trailscontinuously react to input events from the environment. Waiting for an event suspends the running trail until that event occurs. The environment broadcasts an occurring event to all active trails, which share a single global time reference (the event itself).

To illustrate the concurrent reactive nature of CÉU, the example in Figure 1 executes three trails in parallel through the par statement. The first and second trails wait for different events and change the value of a variable, notifying all changes to the third trail, which continuously shows the current value of the variable.

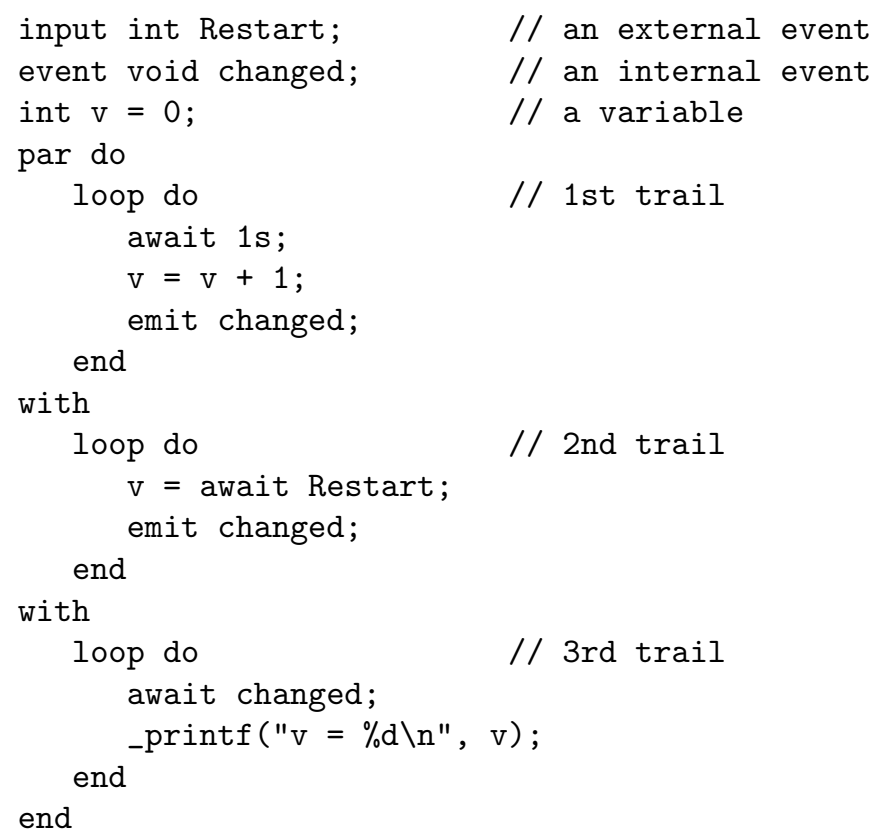

Figure 1: A concurrent program in Céu.

Lines 1-3 declare the variables and events used in the program. An event declaration must include the type of value the occurring event communicates. For instance, the external event Restart carries integer values, while the internal event changed is a notify-only event, holding no values. ${ }_{4}^{4}$

The loop in the first trail (lines 5-9) waits for 1 second, increments variable $v$, and notifies changes through the emit statement (line 8). The loop in the second trail (lines

\footnotetext{
${ }^{4}$ CÉU uses uppercase letters to denote external events and lowercase letters to denote variables and internal events.
} 
11-14) resets $v$ to the value of every occurrence of the input event Restart (line 12), and notifies these changes (line 13). The loop in the third trail (lines 16-19) shows the value of $\mathrm{v}$ (line 18) whenever the event change is emitted (line 17).

CÉU uses a verbose notation with plenty of keywords, such as 'do' and 'end' to delimit blocks (instead of curly brackets). Types, values, and expressions follow the same conventions of $C$. Symbols defined externally in $C$, such as printf in the example, must be prefixed with an underscore to be used in CÉU programs (to be discussed in Section 2.4).

The complete syntax of CÉU is presented in Appendix A.

CÉU is grounded on a precise definition of time as a discrete sequence of external input events $5^{5}$ a sequence because only a single input event is handled at a time; discrete because a complete reaction always executes in bounded time (to be discussed in Section 2.5). The execution model for a CÉU program is as follows:

1. The program initiates the "boot reaction" in a single trail.

2. Active trails execute until they await or terminate. This step is named a reaction chain, and always runs in bounded time.

3. If there are no remaining awaiting trails, the program terminates. Otherwise, the program goes idle and the environment takes control.

4. On the occurrence of a new external input event, the environment awakes the trails awaiting that event. It then goes to step 2.

When multiple trails are active at a time, CÉU does not specify the order in which they should execute. The language runtime is allowed to serialize, interleave, or even parallelize their execution.

If a new external input event occurs while a reaction chain is running (step 2), the environment enqueues it to run in the next reaction, because reaction chains must run to completion.

Every occurring event in CÉU has a corresponding reaction chain that spans for a bounded duration, even if there are no awaiting trails to react to the event. In this case, an empty reaction chain takes place, and the event is discarded.

To illustrate the execution model of CÉU, Figure 2 corresponds to an execution of the program in Figure 3.

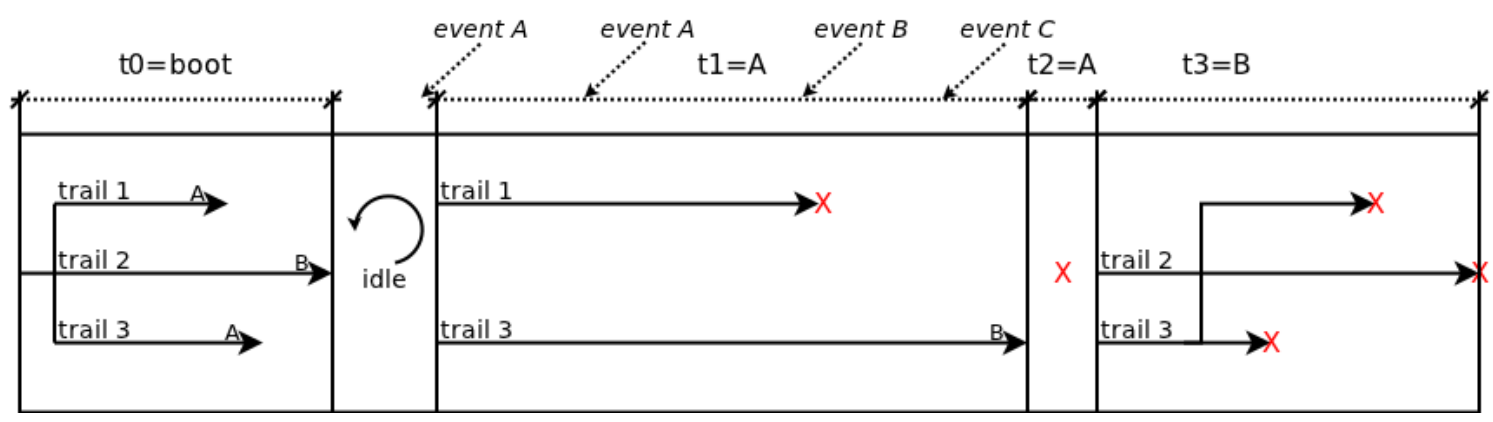

Figure 2: A sequence of reaction chains for the program in Figure 3 .

The program starts in the "boot" reaction in a single trail that splits in three: trails 1 and 3 execute and wait for the event $A$, while trail 2 waits for the event $B$. Control next

\footnotetext{
${ }^{5}$ We use the terms external input event, external event, and input event interchangeably.
} 


\subsection{Internal events \& Dataflow support}

Internal events are used as the single signaling mechanism among trails in CÉU. They also bring dataflow support to the language, permitting that programs create dependency relationships among variables.

Suppose in a program we want that any change to variable v1 automatically updates $\mathrm{v} 2$ to $\mathrm{v} 2=\mathrm{v} 1+1$, and that any change to $\mathrm{v} 2$ updates $\mathrm{v} 3$ to $\mathrm{v} 3=\mathrm{v} 2 * 2$. The program in Figure 4 implements the desired behavior.

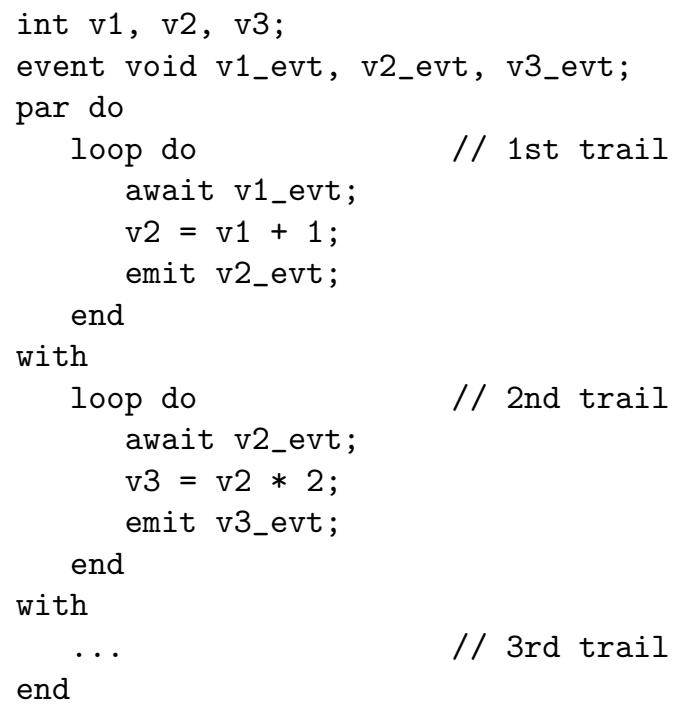

Figure 4: A dataflow program.

We start by defining the variables and corresponding internal events that signal changes (lines 1-2). Any change to a variable in the program must be followed by an emit on the corresponding event so that dependent variables can react. Then, we create two trails to await for changes and update the dependency relations among the variables. For instance, the first trail is a loop (lines 4-8) that waits for changes on v1 (line 5), resets v2 to apply the constraint (line 6), and signals this change (line 7) to make sure that its dependencies are also updated. The behavior for the second trail (lines 10-14), which updates v3 whenever v2 changes, is similar.

In contrast with external events, which are handled in a queue, internal events follow a stack policy and are handled within the same reaction chain. In practical terms, this means that a trail that emits an internal event pauses until all trails awaiting that event completely react to it, continuing to execute afterwards (but still within the same time unit).

In the example, suppose $\mathrm{v} 1$ is updated twice in sequence with the following code in the third trail in parallel (line 16):

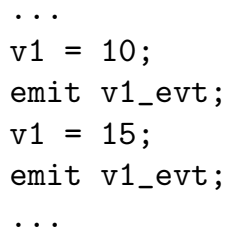


The program behaves as follows (with the stack in emphasis):

1. 3rd trail sets $\mathrm{v} 1=10$, emits v1_evt, and pauses; (stack: [3rd])

2. 1st trail awakes, sets v2 $=11$, emits v2_evt, and pauses; (stack: [3rd,1st])

3. 2nd trail awakes, sets v3=22, emits v3_evt, and pauses; (stack: [3rd,1st,2nd])

4. no trails are awaiting v3 (the event is discarded), so 2nd trail (on top of the stack) resumes, loops, and awaits v2_evt again; (stack: [3rd,1st])

5. 1st trail resumes, loops, and awaits v1_evt again; (stack: [3rd])

6. 3rd trail resumes, sets $\mathrm{v} 1=15$, emits $\mathrm{v} 1$ evt, and pauses; (stack: [3rd])

7. ...

Note that by the time the second "emit v1_evt" executes (step 6), the trails in parallel are already awaiting v1_evt and v2_evt again (steps 4,5); hence, they will react again during the same reaction chain (step 7 on). This behavior, which we consider to be the expected one for emits in sequence, is naturally achieved with a stack execution policy.

An intriguing issue in dataflow languages is when programs have to deal with mutual dependency among variables. Such specifications lead to dependency cycles in programs, which require the explicit placement of delay combinators to break cycles [5].

In CÉU, due to the stacked execution for internal events, such specifications do not lead to runtime cycles. For instance, as we have a finite number of trails, a cycle requires the trail that invoked the first emit to be awaken by the trail that invoked the last emit on the cycle, which is impossible given that the first trail is paused on the emit and cannot be awaiting an event.

As an example, suppose we want to track a temperature in Celsius and Fahrenheit, so that whenever the temperature in one unit is set, the other is automatically recalculated. The program in Figure 5 implements this behavior.

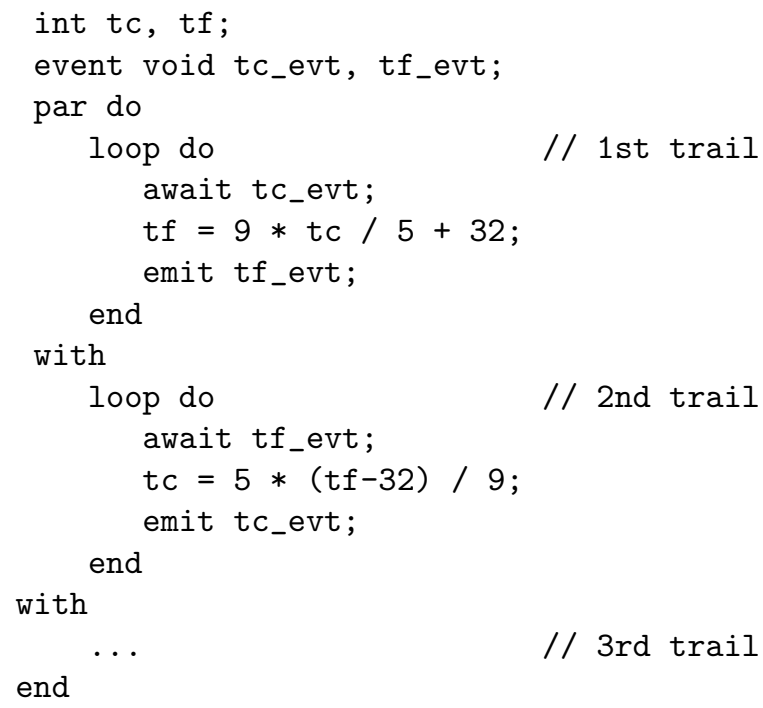

Figure 5: A program with mutual dependency.

Now, consider that the third trail in parallel executes the sequence "tc=0; emit tc_evt": the first trail resumes, updates tf with the conversion formula, emits tf_evt and pauses before awaiting tc_evt again. Then, the second trail resumes, updates tc and emits tc_evt, with no effect on the first trail, which is still paused after emitting tf_evt. Finally, the trails await tc_evt and tf_evt again, and no runtime cycles occur. 


\subsection{Wall-clock time}

Wall-clock tim $\mathrm{g}^{6}$ is probably the most common input in embedded systems, as found in typical patterns like sensor sampling and watchdogs. However, language support for wall-clock time is somewhat low-level, usually through timer callbacks or sleep blocking calls.

For any concrete system implementation, a requested timeout may not expire precisely with zero-delay. We define the difference between the requested timeout and the actual expiring time as the residual delta time (delta). The recurrent use of timed activities in sequence might accumulate a considerable amount of deltas that could lead to incorrect behavior in programs.

CÉU provides an await statement for wall-clock time that handles deltas automatically, resulting in more robust applications. As an example, consider the following program:

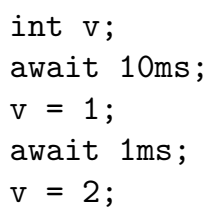

Suppose that after the first await request, the underlying system gets busy and takes $15 \mathrm{~ms}$ to check for expiring awaits. The scheduler will notice that the await $10 \mathrm{~ms}$ has not only already expired, but with delta $=5 \mathrm{~ms}$, and will awake the awaiting trail, which sets $\mathrm{v}=1$ and invokes await 1ms. However, the current delta is higher than the requested timeout $(5 \mathrm{~ms}>1 \mathrm{~ms})$, so the trail is immediately rescheduled for execution, now with delta $=4 \mathrm{~ms}$.

CÉU also takes into account the fact that time is a physical quantity that can be added and compared. For instance, for the following program, if CÉU cannot guarantee that the first trail terminates exactly in $99 \mathrm{~ms}$, it can at least ensure that the program returns 1 :

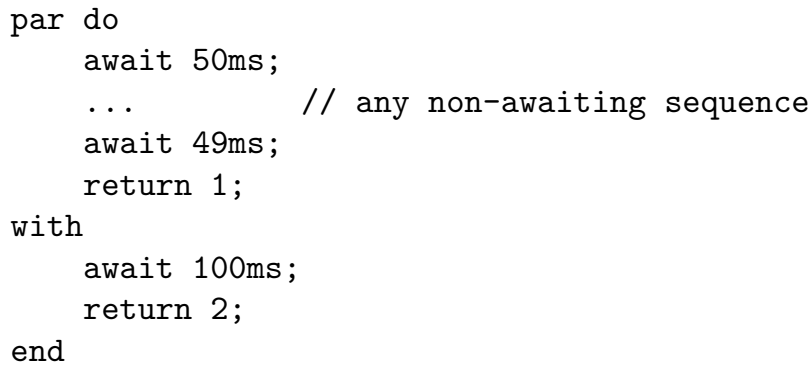

\subsection{Integration with $C$}

The CÉU compiler generates code that is then redirected to the $C$ compiler for the platform in use in order to generate the final binary. Hence, it is important that programs in CÉU have access to all library functions, types, constants, and globals that the underlying $C$ compiler already provides.

Any identifier in a CÉU program prefixed with an underscore is passed $a$ s is to the $C$ compiler (removing the underscore). This way, CÉU programs have access to all global $C$ symbols the target platform offers.

\footnotetext{
${ }^{6}$ By wall-clock time we mean the passage of time from the real world, measured in hours, minutes, etc.
} 
For $C$ calls, CÉU just assumes that they do not loop forever. This responsibility is left to the programmer, and can be easily met by avoiding the use of loops, and blocking/recursive calls. As a general remark, whenever an underscore appears in the code, the programmer must be aware that he is using the " $\mathrm{C}$ hat" and is on his own.

\subsection{Determinism}

Determinism is usually a desired safety property, making concurrent programs predictable and easier to debug.

Concurrency in CÉU is characterized when two or more trails segments execute during the same reaction chain. For instance, in the following example, the assignments run concurrently:

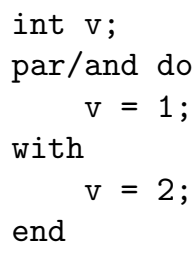

while in

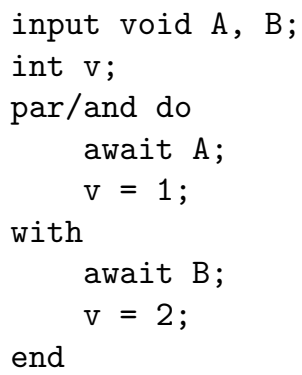

there is no possible concurrency between the assignments, as $A$ and $B$ are external events and cannot happen at the same time (by CÉU's definition of time).

There are three possible sources of nondeterminism in CÉU: concurrent access to variables, concurrent access to internal events, and concurrent $C$ calls.

During compile time, CÉU performs a temporal analysis in order to detect nondeterminism in programs. The static analysis generates a deterministic finite automata that represents a program and covers exactly all possible points it can reach during runtime.

As an example, the DFA in Figure 8 corresponds to the program in Figure 7 . In state $D F A$ \#8 (after six occurrences of $A$ ) the variable $\mathrm{v}$ is accessed concurrently (note the outlined nodes), qualifying a nondeterministic behavior in the program, which is refused at compile time.

If a variable is written in a trail segment, it cannot be read or written in any other concurrent trail segment. For internal events, the reasoning is similar: if an event is emitted, it cannot be awaited or emitted in any other concurrent trail segment. 


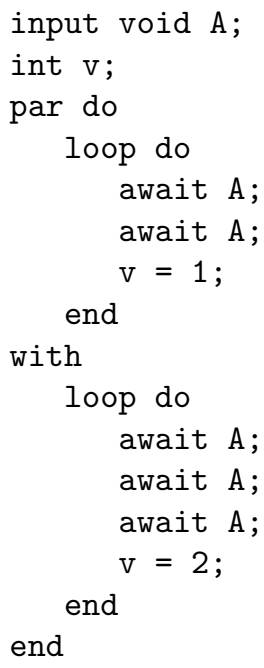

Figure 7: A nondeterministic program.

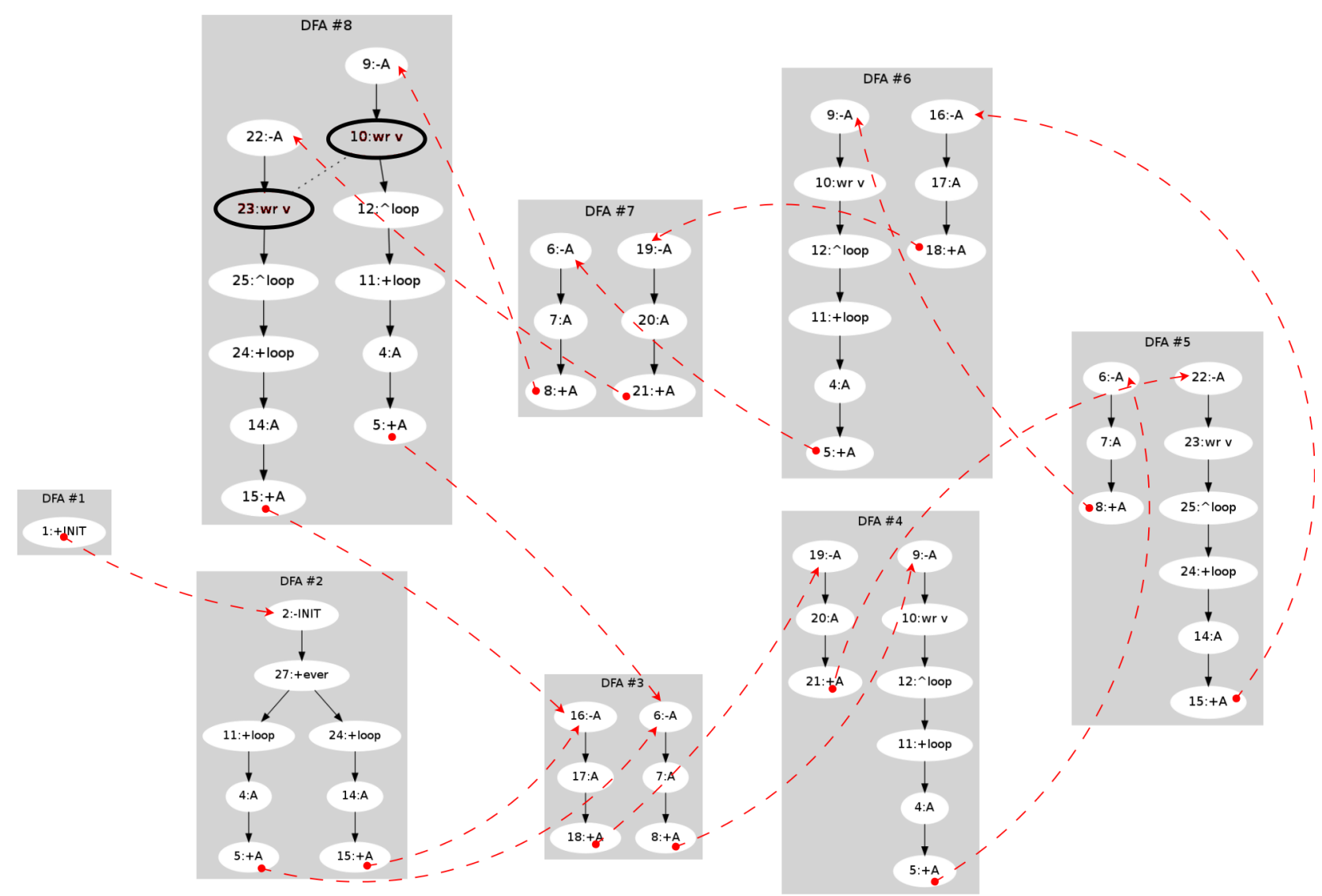

Figure 8: DFA for the nondeterministic example.

Regarding concurrent $C$ calls, CÉU supports annotations that allow specific functions to run concurrently with others. Consider the following program:

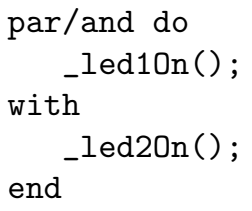


The two calls affect different LEDs, and the order each LED is turned on cannot be perceived in practice. Nonetheless, CÉU is strict about determinism and refuses this program by default.

The pure modifier of CÉU specifies functions that may run concurrently with any other function in the program, while the deterministic modifier specifies sets of functions that may run concurrently among each other.

For instance, in the following code:

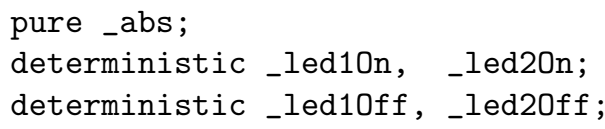

The function _abs may run concurrently with any other functions, while _led10n/_led20n and _led10ff/_led20ff may run concurrently among them.

Finally, the temporal analysis of CÉU also embraces the semantics for wall-clock time. The program

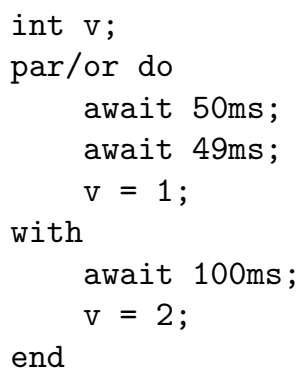

is deterministic, while the program

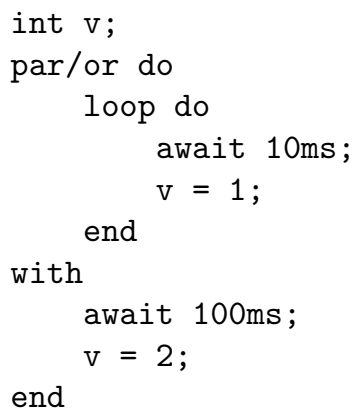

is nondeterministic, as the variable $\mathrm{v}$ is accessed concurrently every ten iterations of the first trail.

Note that CÉU may refuse some deterministic programs (the so called false positives). For instance, the following program is deterministic, but is recognized as nondeterministic by CÉU:

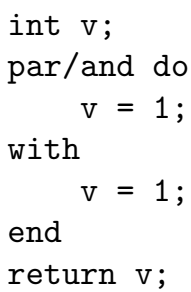

Programs that access the same variables concurrently are always detected as nondeterministic, regardless of the values being assigned or read. 


\subsection{Asynchronous execution}

The main limitation of the synchronous execution model is its inability to perform long computations requiring unbounded loops. Asynchronous blocks fill this gap in CÉU and can contain unbounded loops that run asynchronously with the rest of the program (referred to as the synchronous side).

The program in Figure 9 returns the factorial of 10. The loop (lines 4-11) must be inside the async (lines 2-12) as it contains no await statements. The return statement (line 6) terminates the asynchronous execution setting the variable ret (line 2). We use a watchdog in the enclosing par/or to cancel the computation if it takes longer than $10 \mathrm{~ms}$ (line 15).

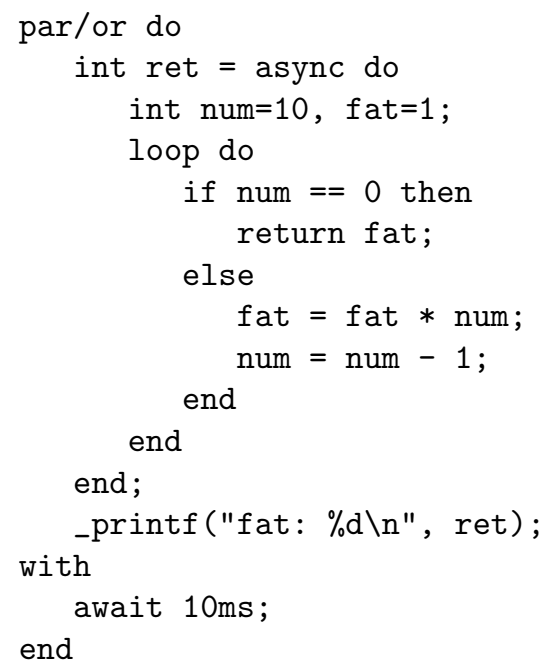

Figure 9: A program with a long computation.

CÉU specifies that asynchronous code cannot execute when there are pending input events in the synchronous side, which always has higher priority. It gives no warranty that an async will ever terminate. Also, to preserve the disciplined synchronous semantics of CÉU, asynchronous blocks cannot use parallel blocks, cannot await input events, cannot manipulate internal events, and cannot assign to variables defined in outer blocks.

From the synchronous perspective, an async can be thought as an external process that generates an input event back into the program when it terminates. The following code express this idea:

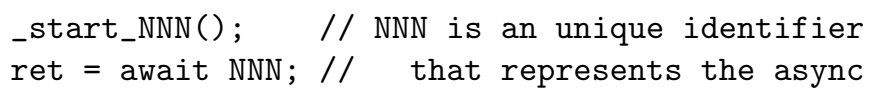

The call to _start_NNN() requests the asynchronous computation to start, while the subsequent "await NNN" resumes when the computation terminates, yielding its final result.

This equivalence emphasizes that asynchronous blocks have a localized impact on the synchronous side of programs (to be discussed in Section 2.9). 


\subsection{Simulation in CÉU}

Simulation is an important aspect in cross-compiling platforms, such as embedded systems. It is usually employed to test applications before deploying them on the target platform. However, simulators are usually inaccurate, may require additional knowledge to operate, and vary among different developing platforms.

CÉU can simulate programs in the language itself, not depending on any external tool to test its programs: asynchronous blocks are allowed to emit input events and also events that represent the passage of wall-clock time towards the synchronous side of the program. This way, it is easy to simulate and test the execution of programs with total control and accuracy with regard to the order of input events-all is done with the same language and inside the programs themselves.

Note that in a reactive language, a program execution depends solely on the events it receives from the environment. Also, in a deterministic program, the exact timings for the incoming events are irrelevant to the application outcome, only the order they arrive.

Suppose we want to simulate the execution of the program in Figure 10, which initially awaits the input event Start and then increments $v$ every 10 minutes during 1 hour and 35 minutes.

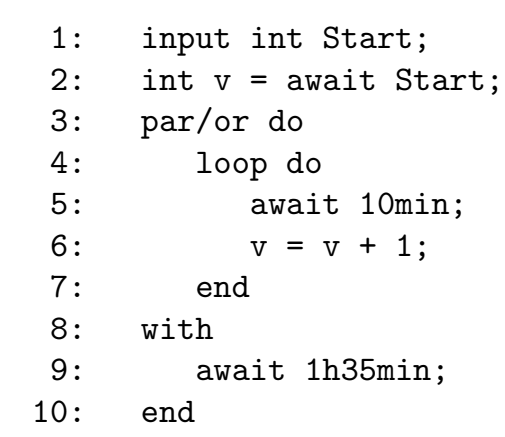

Figure 10: The program to be simulated.

To test this code, we simulate the occurrence of the event Start and the passage of $1 \mathrm{~h} 35 \mathrm{~min}$ in a parallel trail, as shown in Figure 11.

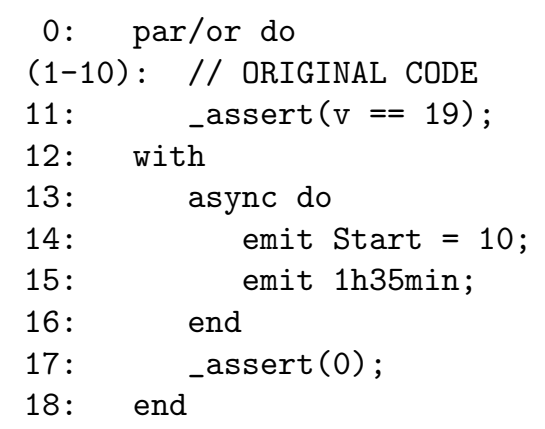

Figure 11: A program that embeds the code in Figure 10 and simulates it. 
The sequence of execution for the simulation will be as follows:

1. The original code (lines 1-10) executes before the async and initially awaits the event Start (line 2).

2. The async (lines 13-17) begins, emits Start=10 (line 14) and is suspended (the original code takes the priority again).

3. The original code resumes and awaits $10 \mathrm{~min}$ and $1 \mathrm{~h} 35 \mathrm{~min}$ in parallel trails (lines 5 and 9).

4. The async resumes and signals that $1 \mathrm{~h} 35 \mathrm{~min}$ have elapsed (line 15 ).

5. The original code completely reacts to all time: the loop iterates exactly 9 times (lines 4-7) before the trail awaiting $1 \mathrm{~h} 35 \mathrm{~min}$ resumes (line 9) and terminates the innermost par/or. The assertion test on line 11 executes before the one on line 17 (which never executes due to the outermost par/or) and terminates the program successfully.

The original code remains unmodified and is simply pasted into the template that runs the simulation in parallel. With the proper tools, this integration can be made even simpler (e.g. we developed a framework to run tests for the implementation of CÉU with hundreds of programs and test cases defined in separate).

It should be clear from the example that simulation does not test true I/O, only the program behavior given an arbitrary input sequence. For instance, the simulation does not take 1 hour to complete, but actually a negligible time. Also, simulation can be employed-with the exact same behavior-in the developing platform (given CÉU is available) or in the target platform.

\subsection{GALS execution}

CÉU complies with the GALS (globally asynchronous, locally synchronous) model of computation, which states that local activities run synchronized with a common clock, while global activities run with independent clocks. The globally asynchronous part of CÉU is restricted to external input events, $C$ code, and asynchronous blocks, while the locally synchronous part of CÉU extends to all other primitives, such as parallel compositions, variable manipulation, and internal events.

The temporal analysis of CÉU discussed in Section 2.6 ensures that only the locally synchronous part of programs is deterministic. Therefore, CÉU is not an absolutely deterministic language, that is, the behavior of programs may vary from execution to execution.

However, nondeterminism in CÉU is exclusively a consequence of globally asynchronous execution. For instance, the program in Figure 12 is nondeterministic, given that the async runs for an undetermined time, and may terminate before or after the statement await 1s. Even so, the CÉU compiler does not complain about nondeterminism, because the assignments cannot run concurrently.

Note that for simulation purposes, the asynchronous execution can be entirely guided by synchronous code, making programs fully deterministic. For instance, the simulation example of Figure 11 can be repeated many times, with the exact same behavior. 


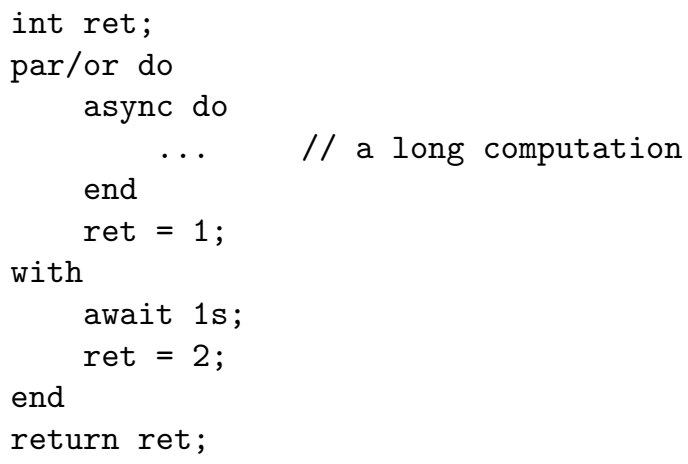

Figure 12: The assignments never run concurrently.

\section{Demo applications}

To demonstrate the expressiveness of CÉU, we implemented three applications in different domains and platforms.

The first example explores Wireless Sensor Networks (WSNs), which are networks composed of a large number of tiny devices (known as "motes") capable of sensing the environment and communicating among them. We integrated CÉU with the TinyOS operating system [6] in order to use the abstracted radio services the operating system provides for motes.

The second example uses the Arduino platform, a popular choice among hobbyists aiming to experiment with electronic components and software. Here, we cannot rely on device drivers and abstract services, as the I/O devices and pin connections vary from application to application. In this context, we make extensive use of thin libraries for specific devices and program directly in the "bare metal".

The third example uses CÉU with the SDL graphics library $7^{7}$ under linux. With a more powerful platform, we can explore some simulation techniques that require fast processing.

The three demos also illustrate different ways to integrate CÉU with an underlying platform.

For TinyOS, we developed a binding that maps all OS services to CÉU. As TinyOS is event-driven, we intercept every possible event it can generate and emit a corresponding external input event in CÉU. The binding is generic and applications can be developed entirely in CÉU.

For Arduino, we do not know in advance which I/O devices are available, hence, it is impossible to provide a unique high-level binding. Instead, we developed a binding that generates events that notify changes on pins used as input ports in the CÉU program.

For SDL, we opted to use the "standalone" binding of CÉU, which starts the application and expects it to generate all input events to itself (inside asynchronous blocks).

The applications are somewhat simple to fit the paper (ranging from 70 to 200 lines), but still complete enough to explore the programming techniques promoted by CÉU.

\footnotetext{
${ }^{7}$ http://www.libsdl.org
} 


\subsection{WSN ring}

In the first demo, we implement a fixed ring topology with three motes placed side by side within their radio ranges ${ }^{8}$

All motes should follow the same behavior: receive a message with an integer counter, show it on the LEDs, wait for 1 second, increment the counter, and forward it to the mote on its right. Note that using fixed topologies and running the same application in all motes are common practices in the context of WSNs.

As the topology constitutes a ring, the counter will be incremented forever while traversing the three motes. If a mote does not receive a message within 5 seconds, it should blink the red LED every 500 milliseconds until a new message is received. In a ring topology, communications traverse all motes, and the network goes down with a failure in a single mote, making tests much easier.

The mote with $i d=0$ is responsible for initiating the process at boot time, and also when the network is down. On perceiving the failure, mote 0 should wait for 10 seconds before retrying the communication.

The code in Figure 13 shows the communicating trail, which receives and forwards the messages forever. The code is an endless loop that first awaits a radio message (line 2 ), gets a pointer to its data region (line 3 ), shows the received counter on the LEDs (line 4), and then awaits 1s (line 5) before incrementing the counter in the message (line 6) and forwarding it to the next mote (line 7).

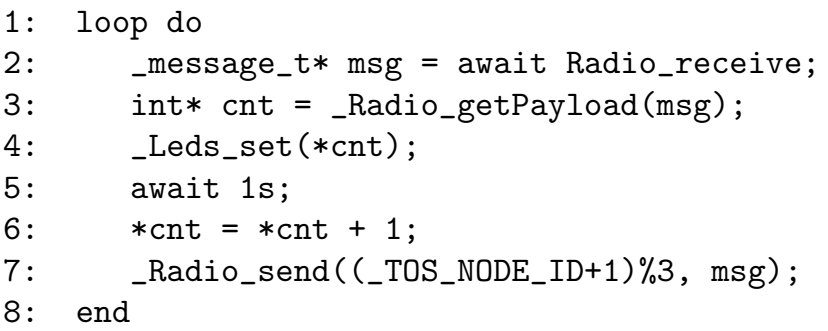

Figure 13: Communicating trail for the ring application.

Because this code does not handle failures, it is straight to the point and easy to follow. Actually, this is the final code for this task, as the task for handling errors is placed in a parallel trail.

Note that the program uses several services provided by the underlying operating system as $C$ functions (LEDs and radio facilities), and none of these calls are blocking.

To handle failures, we use a monitoring trail in parallel with the communicating trail, as Figure 14 shows. The network-down behavior constitutes the lines 12 to 24 . After 5 seconds of inactivity is detected (line 12), two new activities run parallel: one that retries the communication every 10 seconds (lines 14-17) by signaling the internal event retry; and another that blinks the red LED every 500 milliseconds (lines 19-23).

The trick to restore the normal behavior of the network is to await the Radio_receive event (line 26) in a par/or (line 11) with the network-down behavior to kill it whenever the network link is restored. By surrounding everything with a loop (line 10), we ensure that the error detection is continuous.

${ }^{8}$ The complete source code and a video demo for the ring application can be found at http://www. ceu-lang.org/TR/\#ring 


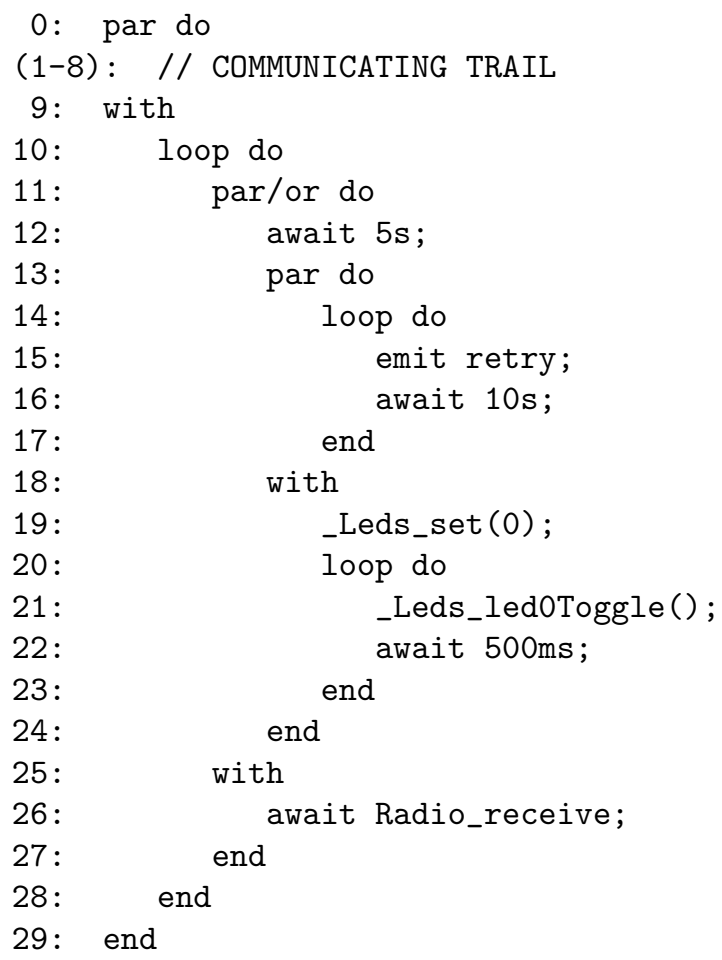

Figure 14: Monitoring trail for the ring application.

Note that both the communicating trail and the monitoring trail waits for the event Radio_receive (lines 2 and 26, respectively), and both react concurrently to it. The first is responsible for handling the message and forwarding it, while the second just kills the network-down behavior (the blinking red LED).

Finally, we need to code the initiating/retrying process that sends the first message from the mote with $i d=0$. As expected we place the code in parallel with the other activities, as Figure 15 shows.

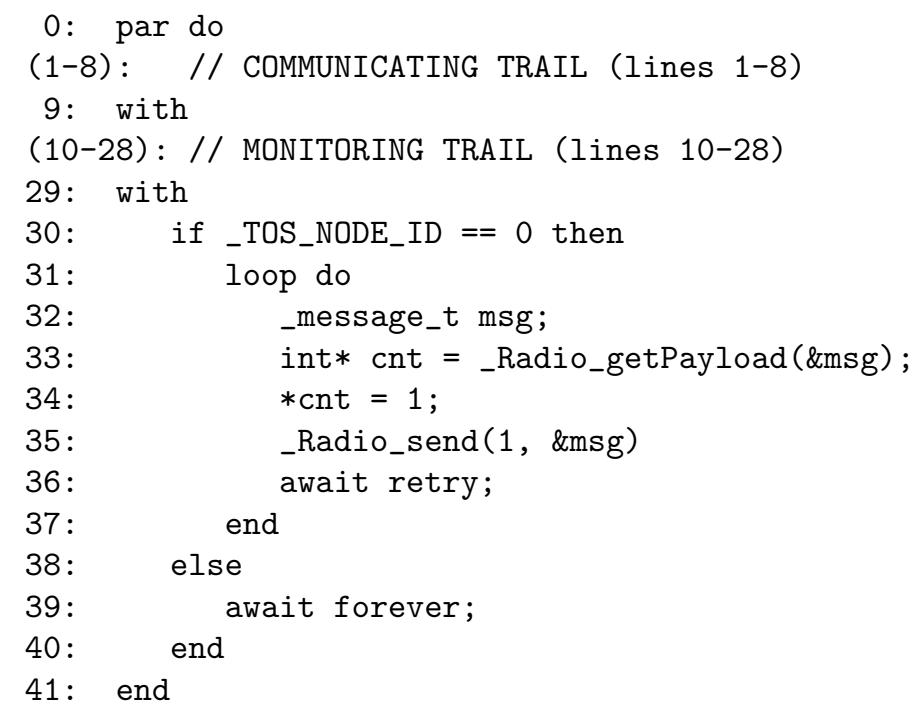

Figure 15: Retrying trail for the ring application. 
We start by checking if the mote has $i d=0$ (line 30). If this is not the case, we simply await forever 19 on this trail (line 39). Otherwise, the loop (lines 31-37) sends the first message as soon as the mote is turned on (line 35). It then waits for a retry emit (line 36) to loop and resend the initial message.

The example shows how complementary activities in an application can be written in separate and need not to be mixed in the code. The activities are then combined together through parallel compositions and communication via internal events to achieve the intended behavior.

The complete source code is less than 70 lines and includes all definitions and code to initialize the radio.

As a final consideration, we can extend the idea of compositions to a new level by combining different applications together. In the context of WSNs, it is usually difficult to physically recover motes in a deployed network, and by combining multiple applications in a single image, we can switch their execution remotely via radio.

The program in Figure 16 illustrates this idea. The input event Switch (line 1) is used to request application switches remotely ${ }^{10}$ Initially, the code behaves as application 1 (lines 7-9), but is also waiting for a Switch request in parallel (line 5). Whenever a new request occurs, the par/or terminates, kills the running application, and restarts as the requested application. The await forever statement (line 13) ensures that a terminating application does not restart by itself.

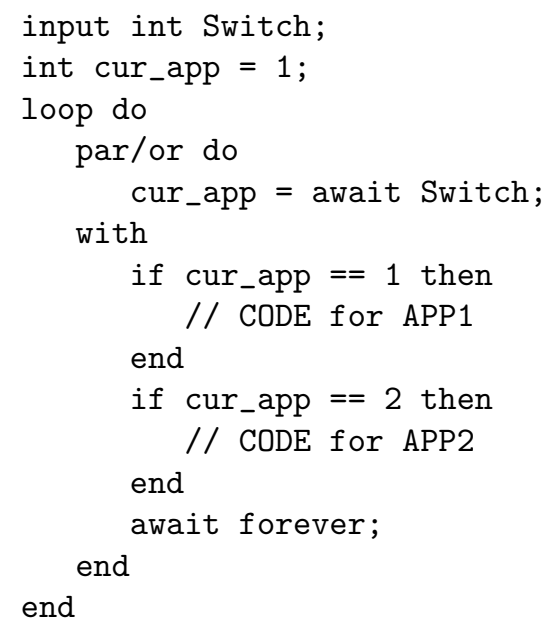

Figure 16: Composition of two applications.

This idea can also be used to reboot a mote remotely, in the case of a strange behavior in an application.

Note that the final ROM image on the mote requires the sum of all installed applications. However, as the applications never execute in parallel, the requirement for RAM is equal to the highest footprint among all installed applications (to be discussed in Section 4.2 .

\footnotetext{
${ }^{9}$ forever is a reserved keyword in CÉU, and represents an external input event that never occurs.

${ }^{10}$ We are assuming the existence of an hypothetical high-level event Switch that abstracts the radio protocol for requests to change the current running application.
} 


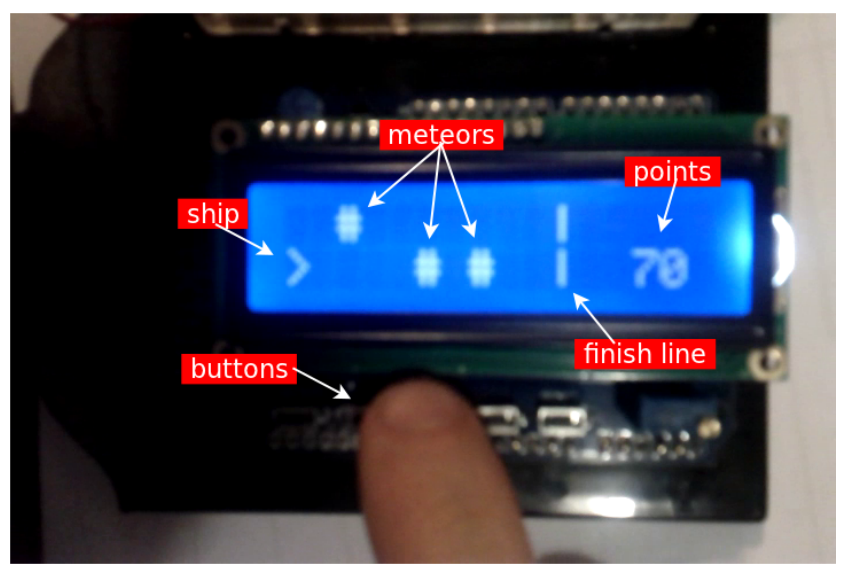

Figure 17: The “ship" game

\subsection{Arduino ship game}

In this demo, we control a ship that moves on space and has to avoid collisions with meteors until reaching a finish line ${ }^{11}$

We use an Arduino connected to a two-row LCD display and two buttons that control the ship. Figure 17 shows the picture of a running quest.

We specify the behavior of the game along with the code and follow a top-down approach. The outermost loop of the game in Figure 18 is responsible for restarting the game every new phase or on "game over". The complete code is constituted of CODE 1 , CODE 2, and CODE 3, which are expanded further.

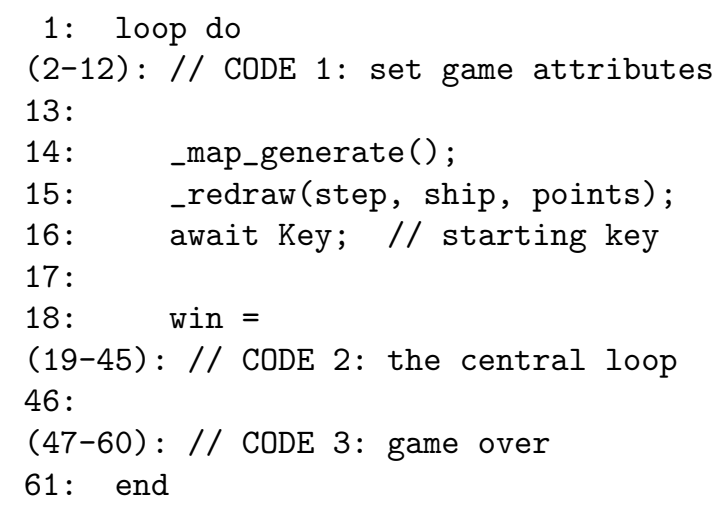

Figure 18: The outermost loop for the ship game.

Every time the loop is executed, it resets the game attributes, such as points and speed (CODE 1, lines 2-12), generates a new map and redraws it on screen (lines 14-15). Then, it waits for a starting key (line 16), and executes the main logic of the game in the central loop (CODE 2, lines 18-45) until the ship reaches the finish line or collides with a meteor. Based on the return status (line 18), the "game over" code (CODE 3, lines 47-60) may display an animation before restarting the game.

The game attributes (CODE 1, in Figure 19) change depending on the result of the previous iteration of the outermost loop.

\footnotetext{
${ }^{11}$ The complete source code and a video demo for the ship application can be found at http://www. ceu-lang.org/TR/\#ship
} 


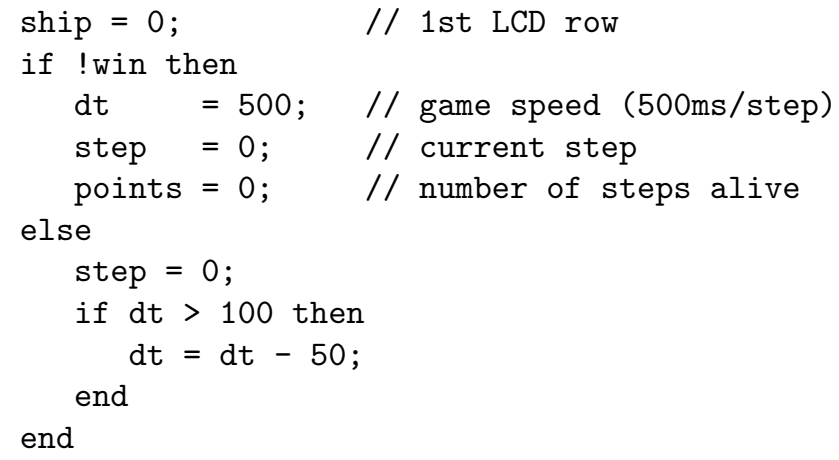

Figure 19: The attributes settings for the ship game.

For the first game execution and whenever the ship collides with a meteor, variable win is set to ${ }^{12}$. hence, the attributes are reset to their initial values (lines 4-6). Otherwise, if the player reached the finish line (win=1), then the game gets faster, keeping the current points (lines 8-11).

The central loop of the game (CODE 2, in Figure 20) is responsible for moving the ship as time elapses and for checking whether the ship reached the finish line or collided with a meteor.

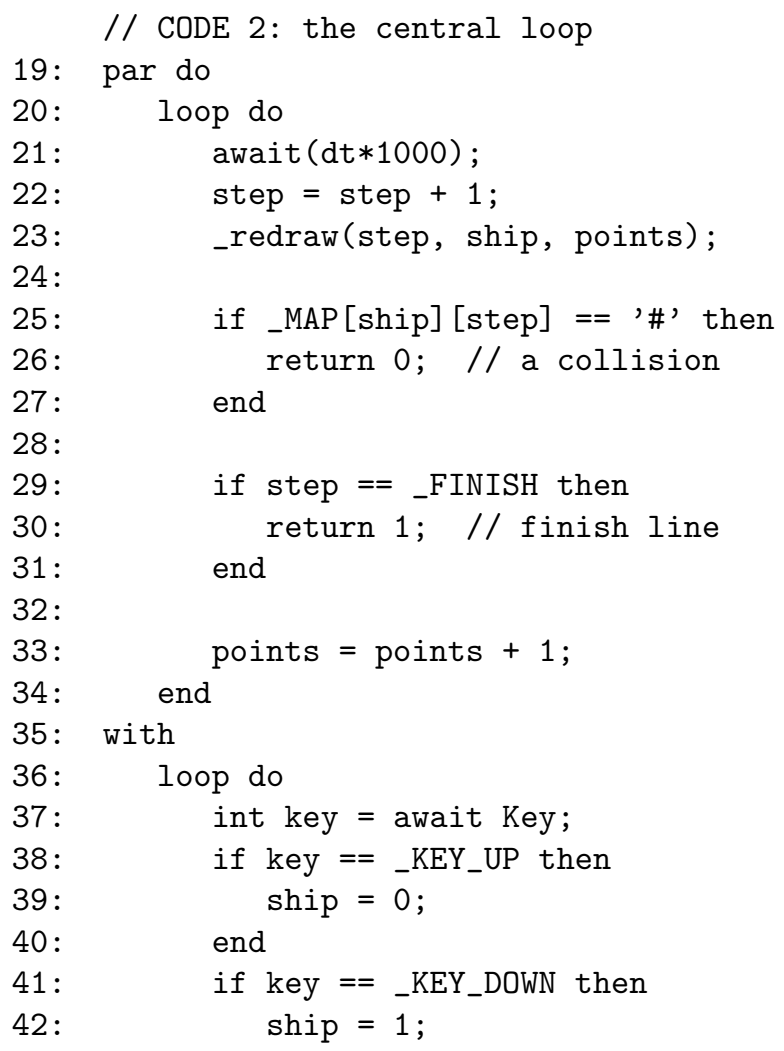

Figure 20: The central loop for the ship game.

\footnotetext{
${ }^{12}$ We omitted all global declarations in the code.
} 
The central loop is actually split in two loops in parallel, one to run the game steps (lines 20-34), and the other to handle input from the player (lines 36-44).

The game steps run periodically, depending on the current speed of the game (line 21). For each loop iteration, the step is incremented and the screen is redrawn (lines 22-23). Then, the ship is checked for collision with meteors (lines 25-27), and with the finish line (lines 29-31). CÉU supports returning from blocks with an assignment, hence, lines 26 and 30 escape the whole par and assign to the win variable in the outer loop (line 18). The points are incremented before each iteration of the loop (line 33).

To handle input events, we wait for key presses in a loop (line 37) and change the ship position accordingly (lines 39, 42). Note that there are no possible race conditions on variable ship because the two loops in the par statement react to different events (i.e. wall-clock time and keys).

After returning from the central loop, we run the code for the "game over" behavior, in Figure 21. which starts an animation if the ship collided with a meteor.

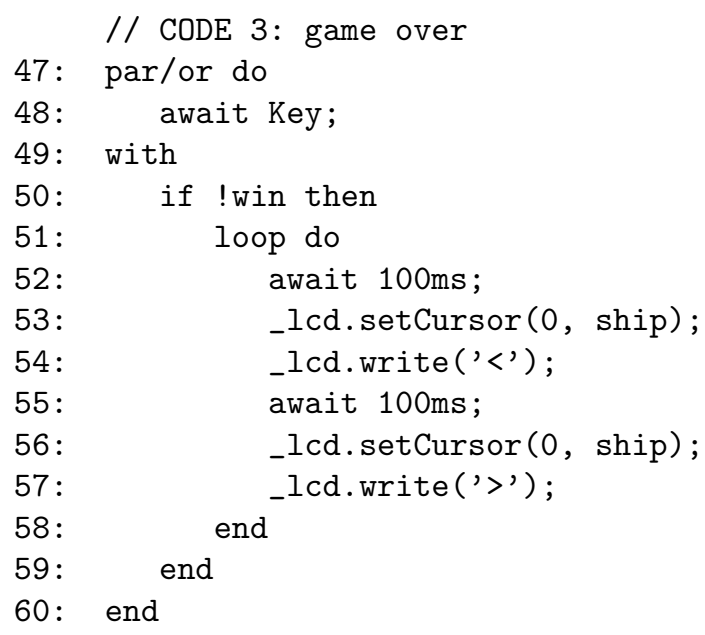

Figure 21: The "game over" code for the ship game.

The animation loop (lines 51-58) continuously displays the ship in the two directions, suggesting that it has hit a meteor. The animation is interrupted when the player presses a key (line 48), proceeding to the game restart.

Finally, we need to generate the key events from the program itself, as we use a thirdparty push-button component not present in all Arduino boards. For this, we place the whole program in parallel with the input event generator, as Figure 22 shows.

The code samples data of an analog port with a delay of $50 \mathrm{~ms}$ to avoid bouncing (lines 65-67). If two consecutive reads point to the same key and they are different from the previous change (line 68), then we change the key (line 69) and generate a new event (in the case of a key press, lines 70-74). The async block is mandatory for generating input events to the program.

The static analysis complains about concurrent $C$ calls of the game code (i.e. map_generate and _redraw) against the event generator code (i.e. _analog2key and _analogRead). By annotating functions with proper modifiers, we get rid of all nondeterministic errors:

pure _analog2key; // just a mapping function deterministic _analogRead with_map_generate, _redraw; 


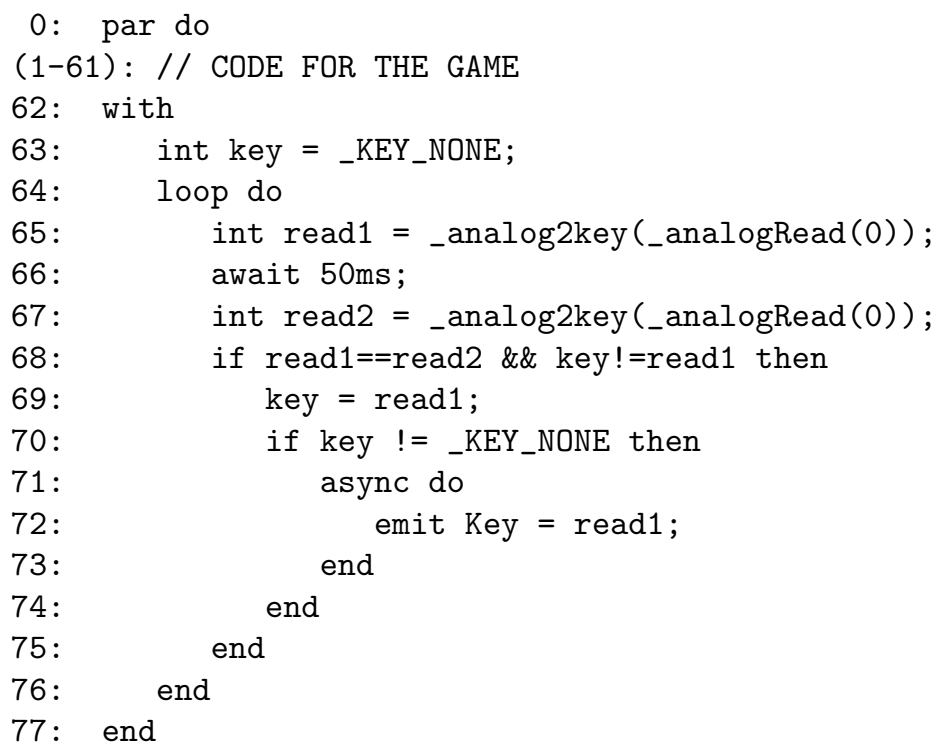

Figure 22: Event generator for the ship game.

The complete source code is around 170 lines and also contains $C$ definitions to generate the map, redraw the scene on the LCD, etc.

\subsection{SDL game simulation}

In the third demo, we implement a simple game to experiment with simulation techniques. Our goal is to show how a self-contained application can be embedded unmodified in an enclosing environment that may re-execute the application in many ways with the same behavior.

In our game ${ }^{13}$, Mario Bros moves in one direction with a constant speed and jumps in reaction to key presses. A turtle moves in the opposite direction randomly. In the case of a collision with the turtle, Mario is thrown back forcedly. The game is intentionally simple as our main objective is to play with simulation. Figure 23 shows the initialization code for the game.

The first three lines specify the game input interface. Seed is an input event that is emitted once to be used in the generation of random numbers. Key is emitted whenever the player presses a key to jump. Step is emitted every $10 \mathrm{~ms}$ and conducts the execution of the game in discrete steps. The internal event collision (line 4) is generated whenever Mario collides with the turtle.

The game starts waiting for the event Seed (line 6), which is expected to be generated by the environment at the beginning of the game. Then, it proceeds to set the initial positions and speeds for the characters. Mario starts on the left side of the screen and initially moves at a constant speed to the right (lines 9-12). The turtle starts on the right side and initially does not move (lines 14-16). The characters are then displayed on the screen (line 18).

The actual game action follows with three loops that run in parallel (lines 19-59 in Figure 24).

\footnotetext{
${ }^{13}$ The source code and video demos can be found at http://www.ceu-lang.org/TR/\#mario
} 


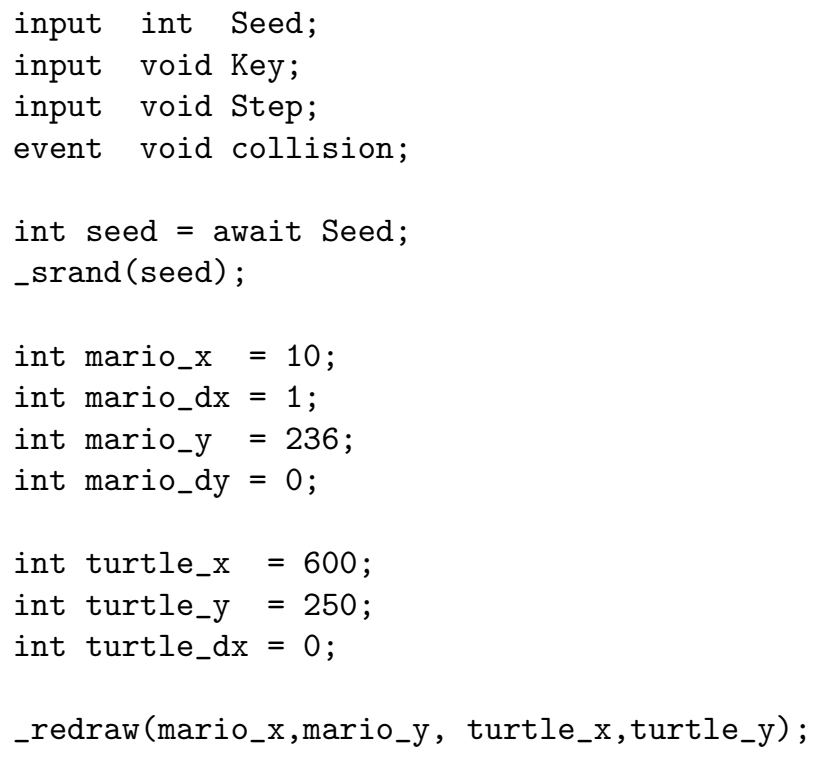

Figure 23: Code for the Mario Bros

The first loop (lines 21-24) randomly changes the turtle speed every 50ms.

The second loop (lines 26-46) is responsible for controlling the speed of Mario, which can be either reacting to a key press (line 29) or to a collision with the turtle (line 32). Whatever happens first is assigned to variable $\mathrm{v}$ (line 27), and the speed of Mario in one of the axis is changed temporarily (lines 35-45). During this period, new key presses and collisions are ignored.

The third loop (lines 48-59) reacts to event Step and is responsible for updating the characters positions (lines 50-52), checking for collisions (lines 53-56), and redrawing the screen (line 57-58).

We embed the presented game code in three different environments. The first variation just provides input for the game (e.g. keys presses and wall-clock time) and does not interfere with it. The second variation also shows the replay of the game after $10 \mathrm{sec}-$ onds of gameplay (in an increased speed). The third variation shows the replay running backwards.

In the remainder of this section, we only discuss the third variation, which encompasses all difficulties found in the other variations.

In order to exhibit the replay of the gameplay, we need to record the input sequence performed by the player and then re-execute the game from scratch by repeating the same input sequence. As discussed in Section 2.8, the behavior of a program in CÉU depends solely on the input order: re-executing a program with the same input must yield the exact same behavior.

However, we want the replay to execute backwards after 10 seconds of gameplay (or 1000 execution steps). For instance, the last scene in the normal execution must be the first one in the replay. To address this requisite, we simulate the passage of all 1000 steps without any delay and without any redrawing of intermediate scenes. Then, we redraw the last scene of the simulation and repeat the whole process, now for one step less (e.g. 9999).

A possible source of nondeterminism in the game are the calls to _srand and _rand 


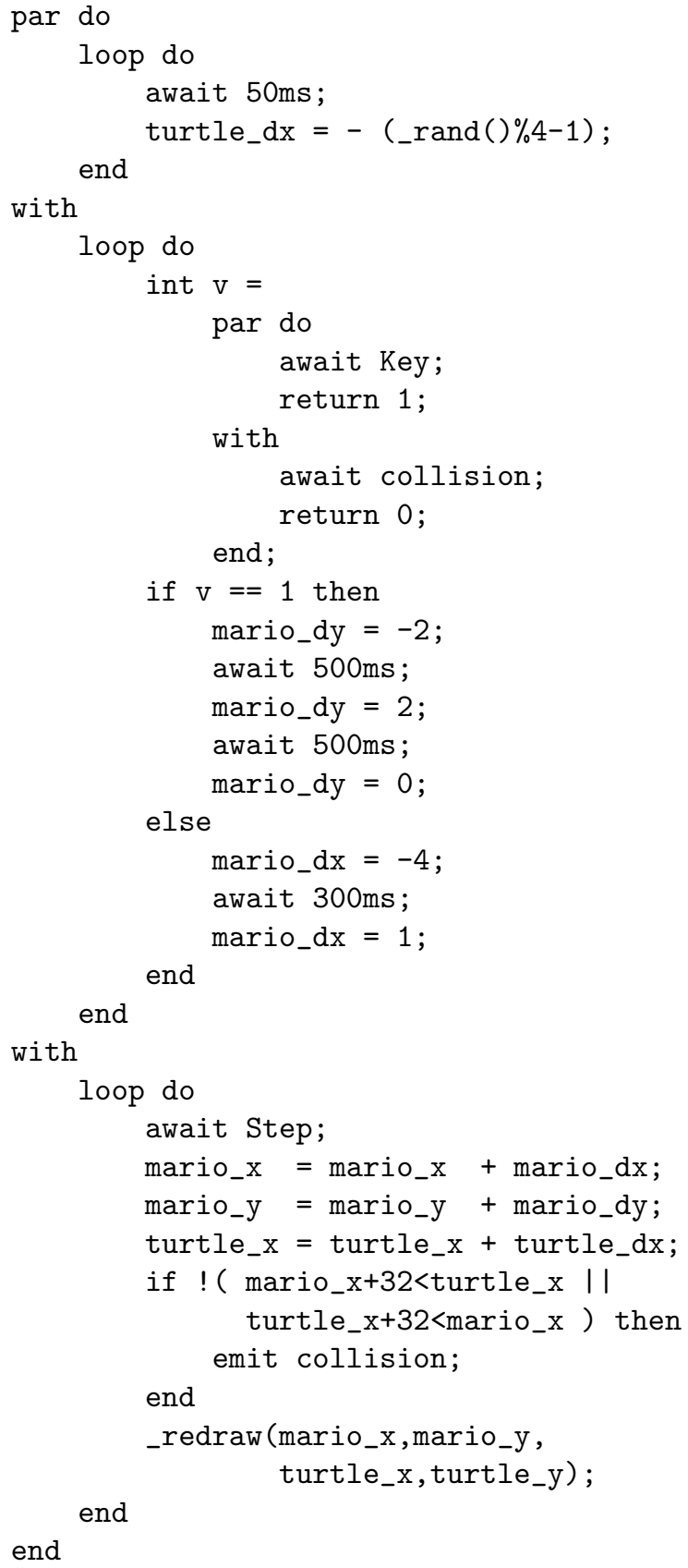

Figure 24: Code for the Mario Bros (cont) 
(lines 7,23), however, they become deterministic when we repeat the seed used in the original execution.

Figure 25 and 26 shows the complete code for the game simulation. As we are not using a preexisting SDL binding for CÉU, we need to emit all events from an async placed in parallel with the game.

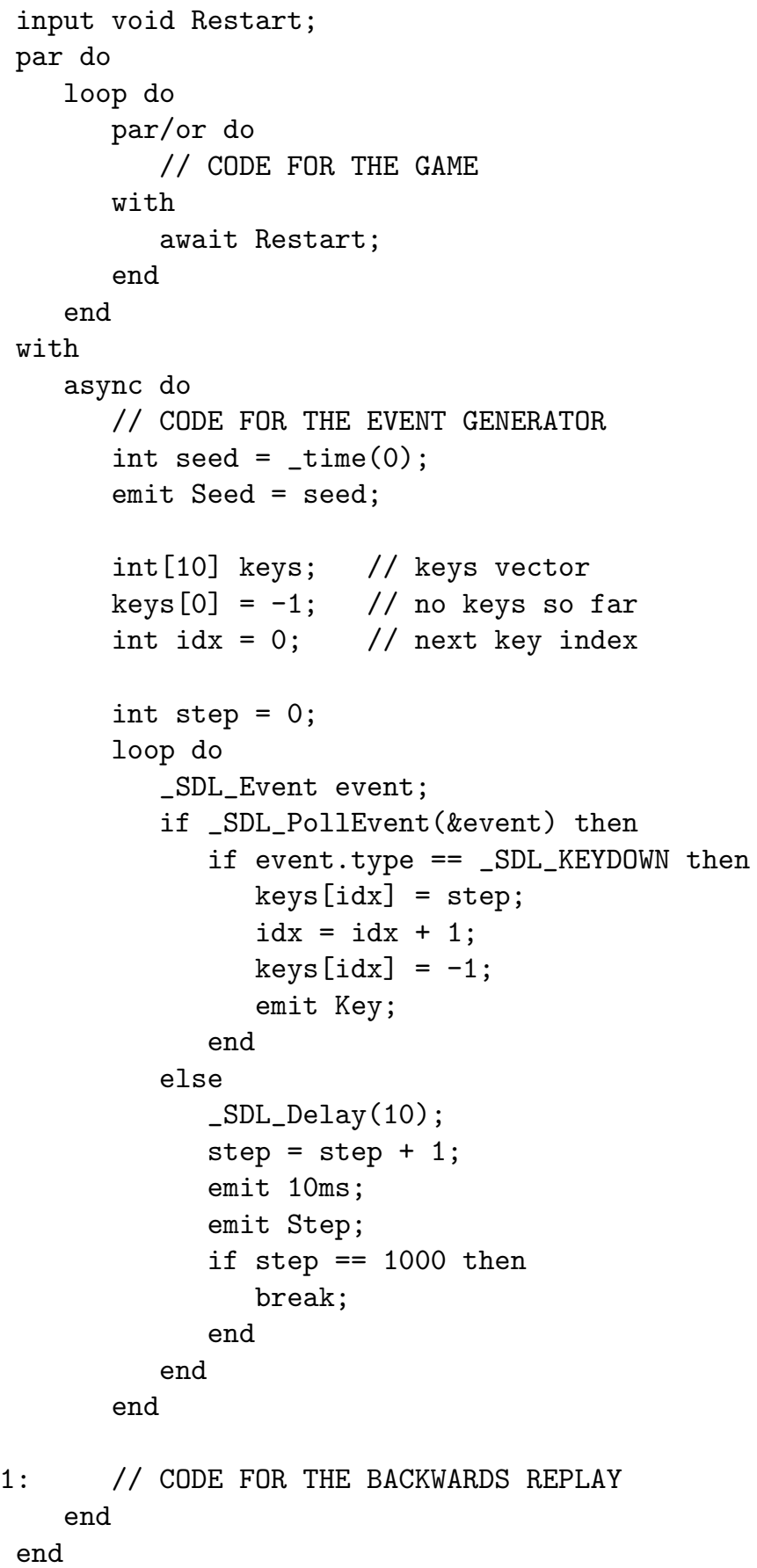

Figure 25: Code for the Mario Bros simulation.

The game code (line 5) runs in parallel with the async responsible for generating events (lines 11-72). Also, we need to be able to restart the game from any point, so we use the event Restart (line 1) as a watchdog running in parallel with the game code (lines 3-9). 


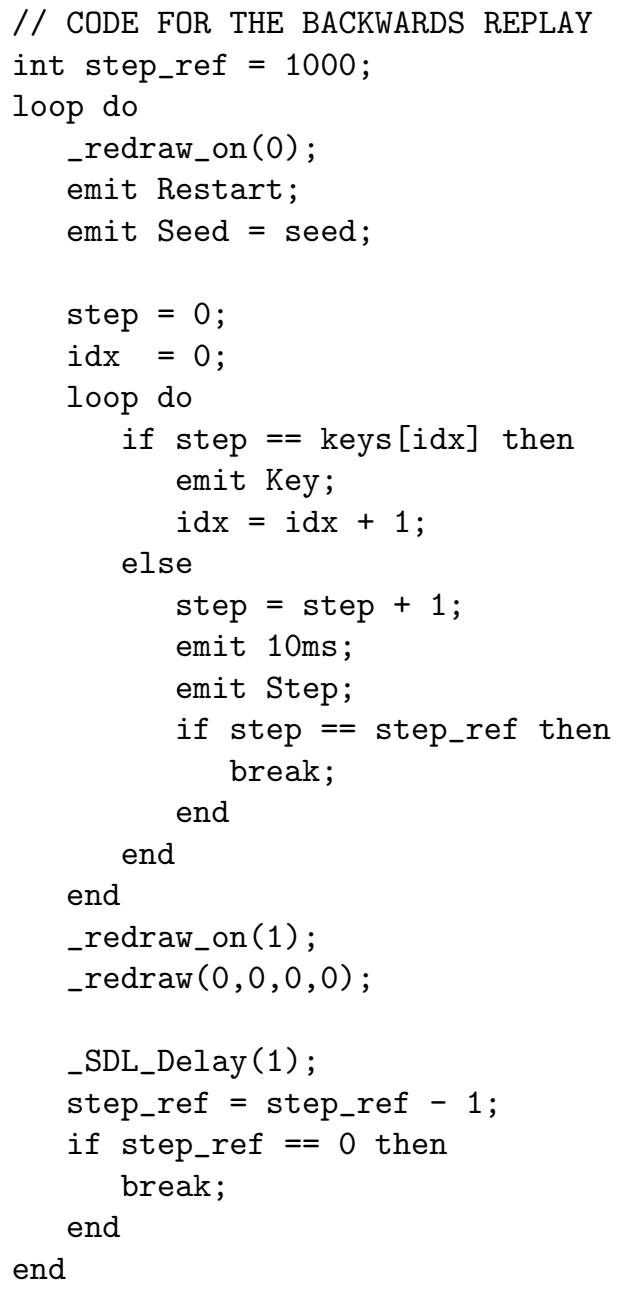

Figure 26: Code for the Mario Bros simulation (cont.).

The event generator starts emitting the random seed, which is saved on variable seed to be reused in the replay (lines 13-14).

We use the vector keys to record all steps in which the player presses a key (lines 1618). Each index on the vector holds the step in which a key was pressed. Then, every time the player presses a key, the current step is written on the vector (lines 25-27). The variable step (line 20) keeps track of the current running Step.

The loop in lines 21-39, continuously polls for key events to emit to the game (lines 22-29), and also emits wall-clock time and the event Step periodically (lines 30-38). After 1000 steps (i.e. 10 seconds), we escape the event generator (lines 35-37) and proceed to the replay code (lines 41-71).

With the original execution recorded, we can now re-execute the game by feeding it with the same input sequence as many times as desired, as Figure 26 shows.

The variable step_ref (line 42) varies from 1000 to 0 and guides the backwards simulation in the outermost loop following it (lines 43-71). For each step_ref, we first call _redraw_on(0) (line 44) to disable redrawing of intermediates scenes. Then, we emit a Restart and the original Seed to the game (lines 45-46) and simulate the execution in the innermost loop up to the current step_ref (lines 50-62). The innermost loop emits a Key event for each step matching the recorded vector (lines 51-53). Also, it simulates the steps 
(lines 55-57), escaping the loop when reaching the current step_ref. After that, the simulation reached the last scene for the current step_ref, so we redraw it on the screen (lines 63-64). Before proceeding to the next outermost loop iteration (i.e. the previous step), we delay the execution to make the replay visible (lines 66-70).

Note that all variables in the code for the game are local to it, hence, they are reinitialized correctly after each Restart. It is fundamental that all side effects in the game code are localized and do not extrapolate it.

In our example, the only exception to this rule is the call to _redraw, which is handled with the specific tweak that enables and disables screen redrawing.

\section{Implementation of CÉU}

As a static language, much of the complexity of the implementation of CÉU resides in the compile phase. Nonetheless, some complexity is left to the runtime phase, which has to handle multiple queues for active trails, asynchronous code, and wall-clock time.

The CÉU parser is written in LPeg [7], and converts a program into an abstract syntax tree (AST) to be used in the following phases.

The program in Figure 27 is used as our guiding example for this section.

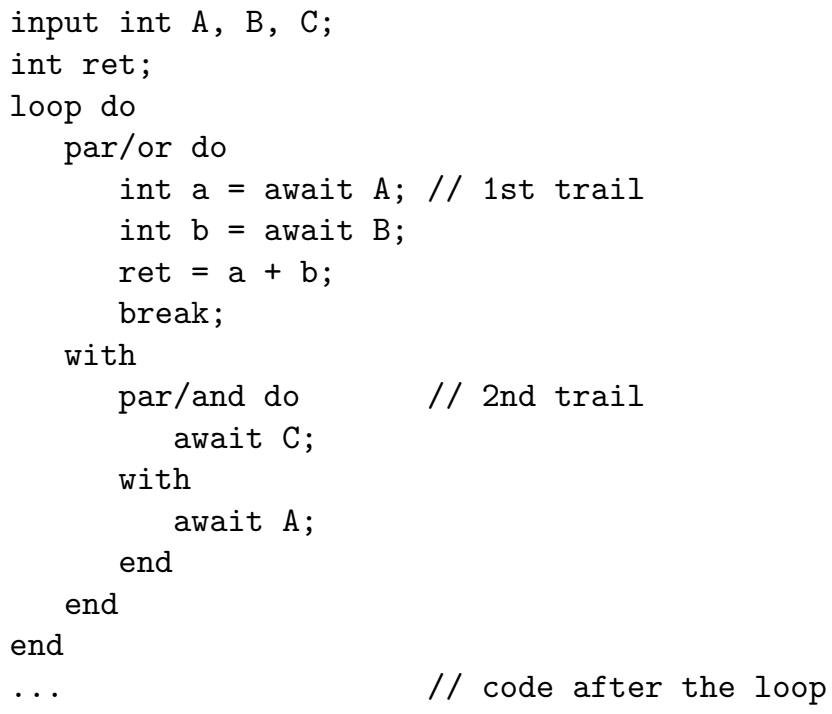

Figure 27: Guiding example for Section 4

\subsection{Temporal analysis}

The temporal analysis phase detects inconsistencies in CÉU programs, such as tight loops and the forms of nondeterminism, as discussed in Sections 2.5 and 2.6. It is also responsible for setting the priorities for trails (see further) and determining the sizes of the queues that are used during runtime.

The program AST is first converted into a graph that represents the execution flow. Figure 28 shows the corresponding graph for our example. 


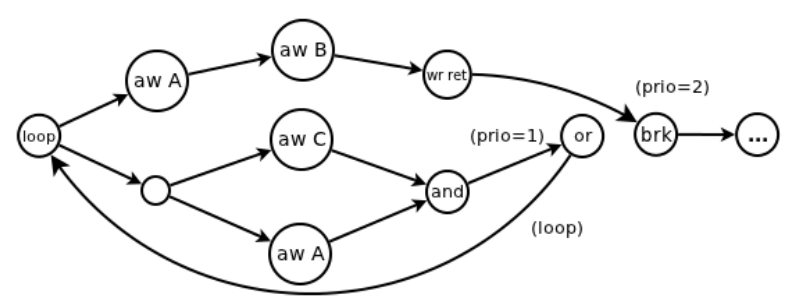

Figure 28: Flow graph for our guiding example

By default, all nodes in a flow graph have priority 0 (highest). However, as the figure shows, nodes that represent the termination of par/ors and loops have lower priorities (the outer, the lower). The priority scheme is needed to avoid glitches during runtime, and is equivalent to traversing a dependency graph in topological order, as employed in functional reactive programming implementations. [5]

The flow graph is then converted to a DFA, as exemplified in Section 2.6

\subsection{Memory layout}

CÉU favors a fine-grained use of trails, being common to use trails that await a single event. For this reason, CÉU does not allocate per-trail stacks; instead, all data resides in fixed memory slots - this is true for the program variables as well as for temporary values and flags needed during runtime. For instance, the first trail in the guiding example requires temporary slots to hold the locals a and $b$, while the second trail must keep flags to remember which sides of the par/and have already terminated.

Memory for trails in parallel must coexist, while statements in sequence can reuse it. In the example, the code following the loop (identified as ...) reuses all memory from the loop.

CÉU statically allocates a one dimension vector to hold all memory slots, whose size is the maximum the program uses at a given time. A given position in the vector may hold different data (with variable sizes) during runtime.

\subsection{Gate allocation}

Each await statement has an associated gate that indicates whether it is currently active (awaiting) or not. Gates for the same event are grouped in a list that is traversed whenever the event occurs, waking the statements whose gates are active. In contrast with memory slots, gates are global and cannot be reused in different parts of the program.

In the example, there is one gate for each of the four await statements. For instance, when the event A occurs, its list of two gates is traversed in order to awake its currently active awaiting trails.

All gates are set to inactive when a program starts. Once an await statement is reached, its corresponding gate is turned on. Once an await statement awakes, its corresponding gate is turned off.

In CÉU, there is a strict relation between gates and trails. A trail can be seen as a sequence of atomic operations with await statements separating them. If a trail is active, it must be awaiting an event. Therefore, a trail can be destroyed by blindly setting all of its gates to inactive. Also, gates in parallel trails use consecutive memory slots, hence, 
destroying trails in parallel is as easy as setting the respective range of gate slots to zero with a memset operation. This is exactly what CÉU does to sibling trails when a par/or or loop terminates.

\subsection{Code generation}

The final output of the CÉU compiler is code in pure $C$, which is not only highly portable across platforms, but also omnipresent in embedded systems. For some CÉU statements, such as calls and expressions, the conversion is straightforward and maps directly to $C$.

The biggest semantic mismatch between $C$ and CÉU resides in the await and parallel statements, which have no analogous in $C$. Consider the follows sequence from the example:

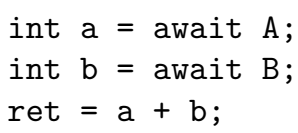

It is clear that before performing the assignment to ret, the program must yield control to the environment twice to await the input events $A$ and $B$. Hence, the generated code must be split in three parts: before awaiting A, before awaiting B, and finally performing the addition and assignment. Figure 29 shows the pseudo-code generated for that sequence.

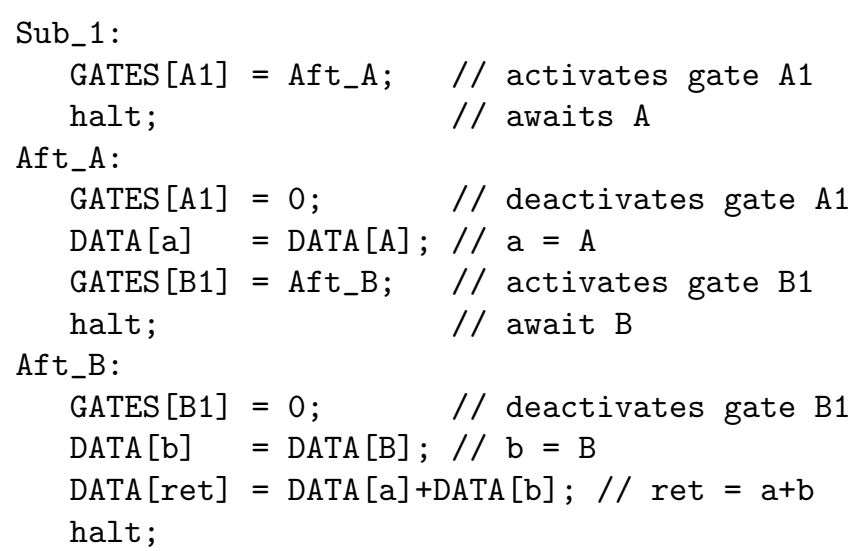

Figure 29: Pseudo-code for a sequence of awaits.

Labels Sub_1, Aft_A, and Aft_B represent entry points into the code, known as tracks, held in gates and which CÉU spawns according to the occurring input event and state of gates. Recall that locals such as a and b cannot be held on the stack, as the halt instruction yields control back to the environment between awaits.

CÉU holds spawned tracks in a queue that is traversed respecting their priorities. This way, a parallel statement simply inserts its tracks (one for each sub-block) into this queue and halts, letting the scheduler decide when they execute. 
For instance, the par/or in the example spawns the track Sub_1 of the previous chunk:

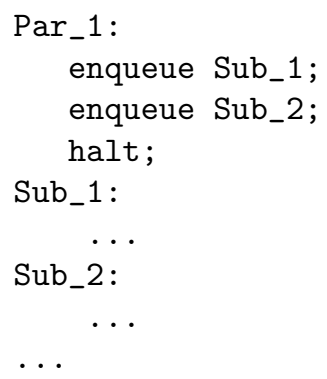

In the final code, illustrated in Figure 30 , the track labels become $C$ switch case labels, which are all enclosed by a loop that traverses the queue of spawned tracks (Q_TRACKS).

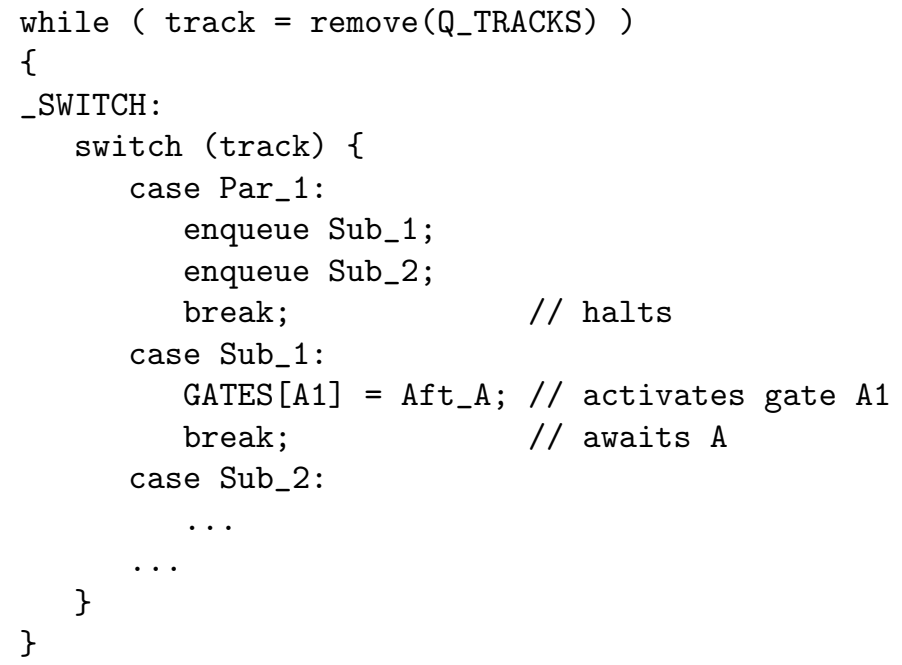

Figure 30: Traversal of the queue for tracks.

Note the _SWITCH goto label, which is used for control-flow statements (i.e. loops and conditionals): in our example, the track Aft B must escape the loop after the assignment. Its actual code is as follows:

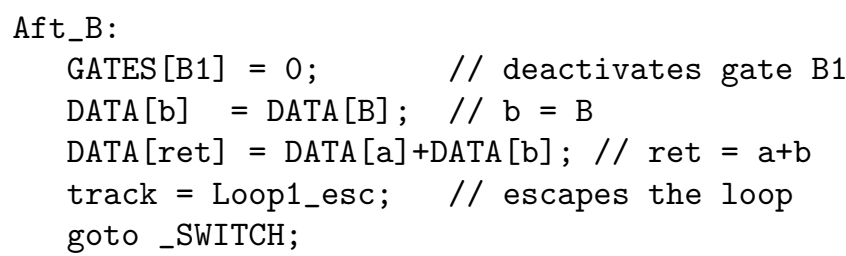

As the code suggests, all tracks execute atomically. This way, even if the temporal analysis is turned off, there are no possible race conditions on shared variables. A possible CÉU implementation exploring parallelism must ensure atomicity among tracks sharing state.

\subsection{Reactive execution}

As a reactive language, the execution of a program in CÉU is guided by the occurrence of external events. From the implementation perspective, there are four external sources of input into programs, which are all exposed as functions in a $C$ API: 
ceu_go_init: initializes internal state (e.g. queues and gates) and executes the "boot" reaction.

ceu_go_event: executes the reaction for the received input id and associated data.

ceu_go_time: receives the current wall-clock time and checks for timeouts (running a reaction if needed).

ceu_go_async: executes a single loop iteration for the next async, switching among them in a round robin policy.

The functions take a bounded time to execute and represent a reaction chain in CÉU. They also return a status code that says if the CÉU program has terminated after reacting to it. Further calls to the API have no effect on terminated programs.

Note that CÉU code running from a call to ceu_go_async may emit an input event or the passage of time. In this case, the $C$ implementation makes a tail call to the corresponding handler (i.e. ceu_go_event or ceu_go_time), as synchronous code has higher priority.

The API reflects the global asynchronous part of CÉU, as discussed in Section 2.9. A simple and opaque API hides local state from the environment, suggesting that the execution varies entirely according to the sequence (and parameters) of API calls.

The bindings for the specific platforms are responsible for calling the functions in the API in the order that better suit their requirements. As an example, it is possible to set different priorities for events that occur concurrently (i.e. while a reaction chain is running). However, a binding must never interleave or run multiple of these functions in parallel. This would break the CÉU sequential/discrete semantics of time, as discussed in Section 2 .

\subsection{Evaluation}

In order to evaluate the current implementation of CÉU, we performed initial experiments in the domain of Wireless Sensor Networks ${ }^{14}$ Our goal is to compare CÉU with other languages implementations regarding two important aspects for WSNs: memory usage and responsiveness 15

\section{Memory usage}

In the first experiment, we ported preexisting nesC [8] applications to CÉU. We chose nes $C$ given its popularity in the context of WSNs, and because it is event based, consuming less memory than multithreaded languages. By using preexisting applications in our experiment, we intend not to choose specific scenarios that favor one language or the other.

Table 1 shows the amount of ROM and RAM for the same applications written in nesC and CÉU. The third line for each application shows the difference for a given measure, for example: the Client application written in CÉU uses 3490 more bytes than its nesC counterpart.

Our experiment suggests that as application complexity grows, the memory footprint of CÉU becomes diluted, and the difference in consumption decreases, showing that CÉU is a viable alternative.

\footnotetext{
${ }^{14}$ The complete source code for the evaluation can be found at http://www.ceu-lang.org/TR/\#exp1

${ }^{15}$ Responsiveness is the ability of a system to promptly acknowledge high-priority requests (e.g. radio messages).
} 


\begin{tabular}{|l|r|r|r|}
\hline \multicolumn{2}{|c|}{} & ROM & RAM \\
\hline \hline \multirow{3}{*}{ Blink } & nesC & 2048 bytes & 51 bytes \\
& CÉU & 5882 bytes & 168 bytes \\
& CÉU-nesC & $\mathbf{3 8 3 4}$ & $\mathbf{1 1 7}$ \\
\hline \hline \multirow{3}{*}{ Sense } & nesC & 4366 bytes & 84 bytes \\
& CÉU & 8086 bytes & 195 bytes \\
& CÉU-nesC & $\mathbf{3 7 2 0}$ & $\mathbf{1 1 1}$ \\
\hline \hline \multirow{3}{*}{ Client } & nesC & 11838 bytes & 329 bytes \\
& CÉU & 15328 bytes & 482 bytes \\
& CÉU-nesC & $\mathbf{3 4 9 0}$ & $\mathbf{1 5 3}$ \\
\hline \hline \multirow{3}{*}{ Server } & nesC & 14648 bytes & 373 bytes \\
& CÉU & 15686 bytes & 443 bytes \\
& CÉU-nesC & $\mathbf{1 0 3 8}$ & $\mathbf{7 0}$ \\
\hline
\end{tabular}

Table 1: Céu vs TinyOS: memory usage

\begin{tabular}{|l|l|c|c|}
\hline \multicolumn{2}{|c|}{} & no comp. & 5 loops \\
\hline \hline \multirow{2}{*}{1 sender } & MantisOS & $23.2 \mathrm{~s}$ & $23.3 \mathrm{~s}$ \\
& CÉU & $23.3 \mathrm{~s}$ & $23.3 \mathrm{~s}$ \\
\hline \hline \multirow{2}{*}{2 senders } & MantisOS & $19.8 \mathrm{~s}$ & $19.9 \mathrm{~s}$ \\
& CÉU & $12.3 \mathrm{~s}$ & $12.4 \mathrm{~s}$ \\
\hline \multicolumn{2}{|l}{ (the measures are the average of three consecutive executions) }
\end{tabular}

Table 2: Céu vs MantisOS: responsiveness

\section{Responsiveness}

In the second experiment, we measure how fast motes can answer radio requests when subjected to long computations. We chose to compare CÉU with MantisOS [9], given that multithreaded systems perform better in this aspect [10]. Table 2 summarizes the results of this experiment, which is described next.

Initially, we created two simple applications that send and receive radio messageswith no processing in parallel - to measure how fast they exchange 3000 messages without losses. We varied the sending speed, and the fastest the receiving side could sustain without losses was around $7 \mathrm{~ms}$ for each message (coincidently, in both implementations), resulting in 23 s for the entire process ("1 sender/no comp." in Table2).

In order to evaluate the responsiveness of the receiving side, we changed it to also execute in parallel five infinite loops that run forever (to represent long computations). In both CÉU and MantisOS implementations, the 3000 messages were still received without losses, while the increase in the total receiving time was negligible (" 1 sender/5 loops" in Table 2).

In MantisOS, we had to change the priority of the receiving thread to be higher than the others. In CÉU the receiving part (which is synchronous) already runs with higher priority than long computations (which run inside asyncs).

In another test, we kept the single receiver and used two senders to measure how fast the receiving side receives 3000 messages (now ignoring the losses) while running long computations in parallel.

Although CÉU performs better than MantisOS (probably due to TinyOS higher performance), our objective is to measure the increase in the total time due to the long com- 
putations running in parallel. Again the increase in time is negligible in both implementations. ("2 senders" in Table2).

From the second experiment, we conclude that CÉU is comparable to a multithreaded implementation in terms of responsiveness, both having nearly optimal behavior for the tests we performed. Although not in the scope of this work, we asserted that, for all tests, both implementations performed a fair scheduling among long computations.

\section{Related work}

Programming languages can be generically classified in two major execution models.

In the asynchronous model, the program activities (e.g. threads and processes) run independently of one another as result of nondeterministic preemptive scheduling. In order to coordinate at specific points, these activities require explicit use of synchronization primitives (e.g. mutual exclusion and message passing).

In the synchronous model, the program activities (e.g. coroutines and CÉU trails) require explicit scheduling primitives (e.g. yield and CÉU await). For this reason, they are inherently synchronized, as the programmer himself specifies when they should execute.

We use this classification to give an abstract overview of related works in this section, although we also comment about specific languages and systems in our discussion.

Note that the terms synchronous and asynchronous are somewhat ambiguous, as they may be used in different contexts. The reason is that synchronous languages require asynchronous primitives (i.e. nonblocking calls), while asynchronous languages require synchronous primitives (e.g. locks and semaphores). We use the definition of synchronous languages as found in [1, 11].

\subsection{Synchronous model}

In this section, we present a review of some synchronous languages and techniques that relate to CÉU.

\section{Event-driven programming}

At the lowest abstract level of the synchronous model, event-driven programming is usually employed as a technique in general-purpose languages with no specific support for reactivity. Because a single line of execution and stack are available, programmers need to deal with the burden of manual stack management and inversion of control. [4]

In the context of embedded systems, the programming language nes $C[8]$ offers eventdriven programming for the TinyOS operating system. The concurrency model of nesC is very flexible, supporting the traditional serialization among callbacks, and also asynchronous callbacks that interrupt others. To deal with race conditions, nes $C$ supports atomic sections with a similar semantics to mutual exclusion in asynchronous languages. We use nesC as the back end of CÉU for TinyOS.

\section{Cooperative multithreading}

Cooperative multithreading is an alternative approach to preemptive multithreading where the programmer is responsible for scheduling activities in the program (known as coroutines [12] in this context). With this approach, there are no possible race conditions on global variables, as the points that transfer control in coroutines are explicit (and, 
supposedly, are never inside critical sections).

Protothreads [13] offer very lightweight cooperative multithreading for embedded systems. Its stackless implementation reduces memory consumption but precludes support for local variables. Furthermore, Protothreads provide no safety warranties besides being race-free: a program can loop indefinitely, and access to globals is unrestricted.

Coroutines are similar to CÉU trails, as they both offer multiple sequential lines of execution to handle concurrent activities. However, CÉU's par/and and par/or composition statements offer a powerful abstraction to avoid manual bookkeeping of activities, such as creating, starting, rejoining, and destroying them. Also, the semantics for rejoins in parallel compositions is fundamental for the temporal analysis of CÉU, which cannot be done effectively with coroutines.

\section{Finite state machines}

The use of finite state machines (FSMs) is a classic technique to implement reactive applications, such as network protocols and graphical user interfaces. A contemporary work [14], based on the Statecharts formalism [15], provides a textual FSM language targeting Wireless Sensor Networks.

FSMs have some known limitations. For instance, writing purely sequential flow is tedious, requiring to break programs in multiple states with a single transition connecting each of them. Another inherent problem of FSMs is the state explosion phenomenon. To alleviate this problem, some designs support hierarchical FSMs running in parallel [14]. However, adopting parallelism precludes the use of shared state, or at least requires a static analysis such as that of CÉU.

\section{Dataflow}

Dataflow programming [3, 16] differs from the traditional "Von Neumann" imperative style, where programs are defined as sequences of steps. With a declarative style, dataflow programs define high-level dependency relationships among data. The language is responsible for scheduling activities that propagate external changes into the dependency graph that represents a program.

The Functional Reactive Programming (FRP) paradigm brings dataflow behavior to functional languages [17]. CÉU borrows some ideas from a FRP implementation [5], such as a push-driven evaluation and glitch prevention. Dataflow in CÉU is limited to static relationships, and the way dataflow programs are expressed is less abstract than in FRP.

However, embedded systems are typically characterized by control-intensive applications, where programs have to deal with low-level I/O and handle explicit state. In this context, dataflow programming does not provide proper abstractions, being more suitable for data-intensive applications.

\section{Esterel}

Our work is strongly influenced by the Esterel language [2], which also provides an imperative reactive style with a similar set of parallel compositions.

However, a fundamental distinction exists: in Esterel, the semantics for time is similar to that of digital circuits, where an external clock defines discrete steps in which multiple signals (events in CÉU) can be queried for their presence status.

With such semantics in CÉU, multiple input events could be active at the same time, making its temporal analysis impossible. As a consequence, access to shared state would be nondeterministic, also breaking dataflow support in CÉU. In Esterel, "if a variable is written in a thread, then it can be neither read nor written in any concurrent thread". [18] 
Regarding features that are orthogonal to the distinction regarding events, CÉU supports "wall-clock" time and simulation from asynchronous blocks, while Esterel provides a suspend statement that cannot be easily implemented on top of the existing primitives (and which we are considering to incorporate into CÉU).

\subsection{Asynchronous model}

The asynchronous model of computation can be sub-divided in how independent activities coordinate. In shared memory concurrency, communication is via global state, while synchronization is via mutual exclusion. In message passing, both communication and synchronization happen via exchanging messages.

The default behavior of activities being independent hinders the development of highly synchronized applications. As a practical evidence, we developed a simple application that blinks two LEDs in parallel with different frequencies ${ }^{16}$. We implemented it in CÉU and also in the two asynchronous styles. For shared memory concurrency, we used a multithreaded RTOS17, while for message passing, we used an occam for Arduino [19].

The LEDs should blink together at a specific rate, depending on the chosen blinking frequencies. We tested several combinations of frequencies looking for asynchronism on the implementations ${ }^{18}$ As expected, the LEDs in the two asynchronous implementations lost synchronism after some time of execution. The CÉU implementation remained synchronized for all tests that we have performed.

The implementations are intentionally naive: they just spawn the activities that blink the LEDs in parallel. The behavior for the asynchronous implementations of the blinking application is perfectly valid, as the preemptive execution model does not ensure implicit synchronization among activities. We used timers in the application, but any kind of high frequency input would also behave nondeterministically in asynchronous systems.

Although this application can be implemented correctly with an asynchronous execution model, it circumvents the language style, as timers need to be synchronized in a single thread. Furthermore, it is common to see similar naive blinking examples in official examples of asynchronous systems ${ }^{19}$, suggesting that LEDs are supposed to blink synchronized.

\section{Conclusion}

We presented CÉU, a language targeting embedded systems that unifies imperative and dataflow reactive programming. CÉU is based on a synchronous kernel that provides reactions to events and imperative control primitives.

For dataflow support, CÉU relies on disciplined access to variables together with internal events as a communication mechanism among trails. The stack execution policy for internal events can express nested emits, and also avoids dependency cycles in programs.

\footnotetext{
${ }^{16}$ The complete source code and a video demos for the application can be found at http://www . ceu-lang. org/TR/\#blink

${ }^{17}$ http://www .chibios .org/dokuwiki/doku.php?id=start

${ }^{18}$ We settled at $400 \mathrm{~ms}$ and $1000 \mathrm{~ms}$, but any combination of two non-divisor numbers behaved the same way in our tests.

${ }^{19}$ Example 1 in the RTOS DuinOS v0.3: http://multiplo.org/duinos/wiki. Example 3 in the occam-based Concurrency for Arduino v20110201.1855: http://concurrency.cc/download
} 
CÉU provides a convenient syntax for wall-clock time, which is justified by the recurrent use of timed activities in embedded applications. Furthermore, native support also avoids dealing explicitly with residual delta times in time awaits.

For time-consuming operations, CÉU provides asynchronous blocks that can execute unbounded loops. By also allowing them to emit events towards the program, CÉU supports simulation in the language itself, not depending on external tools to test programs.

In the design of CÉU we favored safety over expressive power, by restricting the language to static capabilities only. This limitation can be considered (to some extent) advantageous for embedded systems, given that CÉU enforces the prevailing discipline in this context.

Although CÉU trails are allowed to share memory, they are completely race free, and no mutual exclusion mechanisms are required. Also, all memory required during execution is allocated previously, at compile time. CÉU does not use heap storage, nor dynamically growing stacks to hold local variables.

We propose a temporal analysis in programs that prevents unresponsiveness and enforces deterministic behavior by default. Although the temporal analysis conversion algorithm is exponential, it is applicable in practice, considering the size of applications in the context of embedded systems.

The three demo applications we presented illustrate the programming techniques of CÉU in two embedded domains (Wireless Sensor Networks and Arduino), and also in standalone mode to explore simulation. The examples show how complementary activities in a program can be written in separate to run in parallel and need not be mixed with the final code. The examples also make recurrent use of $C$ to interact seamlessly with the underlying platforms.

The CÉU runtime requires a small footprint suitable for highly constrained embedded systems. We presented an initial evaluation of our implementation, showing that CÉU is a viable option regarding memory usage and responsiveness in programs. Moreover, we believe that the gains with a safer and higher-level language pays off minor drops in performance.

\section{References}

[1] BENVENISTE, A.; CASPI, P.; EDWARDS, S.; HALBWACHS, N.; LE GUERNIC, P. ; DE SIMONE, R.. The synchronous languages 12 years later. Proceedings of the IEEE, 91:64-83, Jan 2003.

[2] BERRY, G.; GONTHIER, G.. The ESTEREL synchronous programming language: design, semantics, implementation. Science of Computer Programming, 19(2):87152, 1992.

[3] HALBWACHS, N.; CASPI, P.; RAYMOND, P. ; PILAUD, D.. The synchronous data-flow programming language LUSTRE. Proceedings of the IEEE, 79:1305-1320, September 1991.

[4] ADYA, A.; OTHERS. Cooperative task management without manual stack management. In: ATEC '02, p. 289-302, Berkeley, CA, USA, 2002. USENIX Association.

[5] COOPER, G. H.; KRISHNAMURTHI, S.. Embedding dynamic dataflow in a callby-value language. In: 15TH EUROPEAN SYMPOSIUM ON PROGRAMMING, p. 294-308, 2006. LNCS 3924. 
[6] HILL, J.; SZEWCZYK, R.; WOO, A.; HOLLAR, S.; CULLER, D. ; PISTER, K.. System architecture directions for networked sensors. SIGPLAN Notices, 35:93-104, November 2000.

[7] IERUSALIMSCHY, R.. A text pattern-matching tool based on parsing expression grammars. Softw. Pract. Exper., 39:221-258, March 2009.

[8] GAY, D.; WELSH, M.; LEVIS, P.; BREWER, E.; VON BEHREN, R. ; CULLER, D.. The nesC language: A holistic approach to networked embedded systems. In: PLDI'03, p. 1-11, 2003.

[9] BHATTI, S.; CARLSON, J.; DAI, H.; DENG, J.; ROSE, J.; SHETH, A.; SHUCKER, B.; GRUENWALD, C.; TORGERSON, A. ; HAN, R.. MANTIS OS: an embedded multithreaded operating system for wireless micro sensor platforms. Mob. Netw. Appl., 10:563-579, August 2005.

[10] DUFFY, C.; ROEDIG, U.; HERBERT, J. ; SREENAN, C. J.. A comprehensive experimental comparison of event driven and multi-threaded sensor node operating systems. JNW, 3(3):57-70, 2008.

[11] POTOP-BUTUCARU, D.; DE SIMONE, R. ; TALPIN, J.-P.. The synchronous hypothesis and synchronous languages. In: Zurawski, R., editor, EMBEDDED SYSTEMS HANDBOOK. 2005.

[12] MOURA, A. L. D.; IERUSALIMSCHY, R.. Revisiting coroutines. ACM TOPLAS, 31(2):6:1-6:31, Feb. 2009.

[13] DUNKELS, A.; SCHMIDT, O.; VOIGT, T. ; ALI, M.. Protothreads: simplifying event-driven programming of memory-constrained embedded systems. In: PROCEEDINGS OF THE 4TH INTERNATIONAL CONFERENCE ON EMBEDDED NETWORKED SENSOR SYSTEMS, SenSys '06, p. 29-42, New York, NY, USA, 2006. ACM.

[14] KASTEN, O.; RMER, K.. Beyond event handlers: Programming wireless sensors with attributed state machines. In: THE FOURTH INTERNATIONAL CONFERENCE ON INFORMATION PROCESSING IN SENSOR NETWORKS (IPSN), p. 4552, Los Angeles, USA, Apr. 2005.

[15] HAREL, D.. Statecharts: A visual formalism for complex systems. Science of Computer Programming, 8(3):231-274, June 1987.

[16] ASHCROFT, E. A.; WADGE, W. W.. Lucid, a nonprocedural language with iteration. Communications of the ACM, 20(7):519-526, 1977.

[17] WAN, Z.; HUDAK, P.. Functional reactive programming from first principles. SIGPLAN Notices, 35(5):242-252, 2000.

[18] BERRY, G.. The Esterel-V5 Language Primer. CMA and Inria, Sophia-Antipolis, France, June 2000. Version 5.10, Release 2.0.

[19] JACOBSEN, C. L.; JADUD, M. C.; KILIC, O. ; SAMPSON, A. T.. Concurrent eventdriven programming in occam-pi for the Arduino. In: COMMUNICATING PROCESS ARCHITECTURES 2011, volumen 68 de Concurrent Systems Engineering, p. 177-193, Amsterdam, The Netherlands, June 2011. WoTUG, IOS Press. 


\section{A Syntax of CÉU}

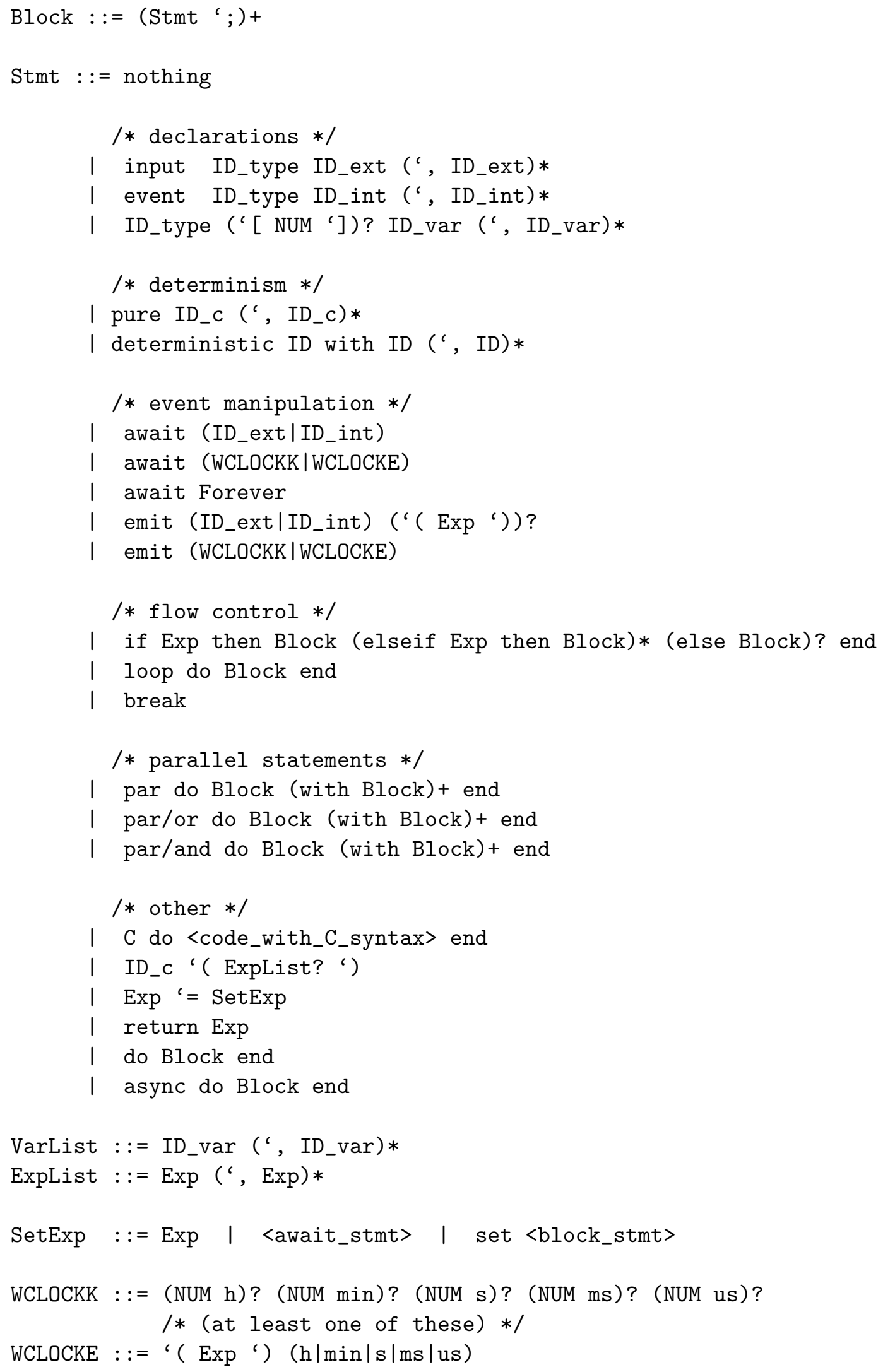


